Supporting Information

\title{
A new access to dihydrotropones through ring expansion of spirocyclohexadienones: Synthesis and mechanism
}

Marie Varin, Angèle Chiaroni, Jean-Yves Lallemand, Bogdan Iorga, Catherine Guillou Institut de Chimie des Substances Naturelles, CNRS UPR 2301, F-91198 Gif sur Yvette, France

Table of contents :

${ }^{1} \mathrm{H}$ and ${ }^{13} \mathrm{C}$-NMR spectra of compound 3

${ }^{1} \mathrm{H}$ and ${ }^{13} \mathrm{C}$-NMR spectra of compound 2a

${ }^{1} \mathrm{H}$ and ${ }^{13} \mathrm{C}$-NMR spectra of compound 13a

Evolution of the reaction followed by ${ }^{1} \mathrm{H}-\mathrm{NMR} \quad S 8$

X-ray structure analysis of compound 2a $\quad S 11$

$\begin{array}{ll}\text { Theoretical data for intermediates and transition states } & \text { S19 }\end{array}$ 


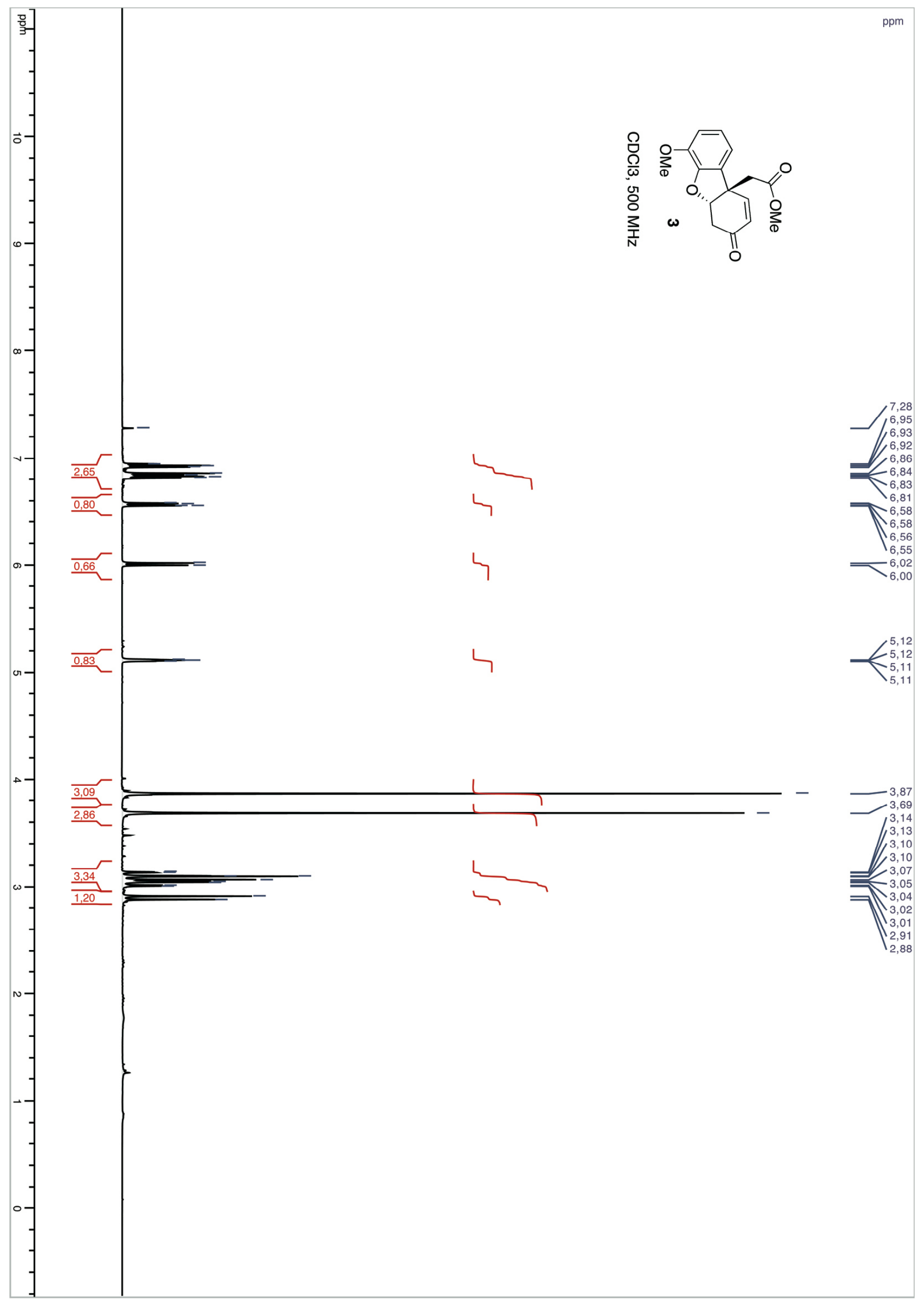




$$
\mid
$$




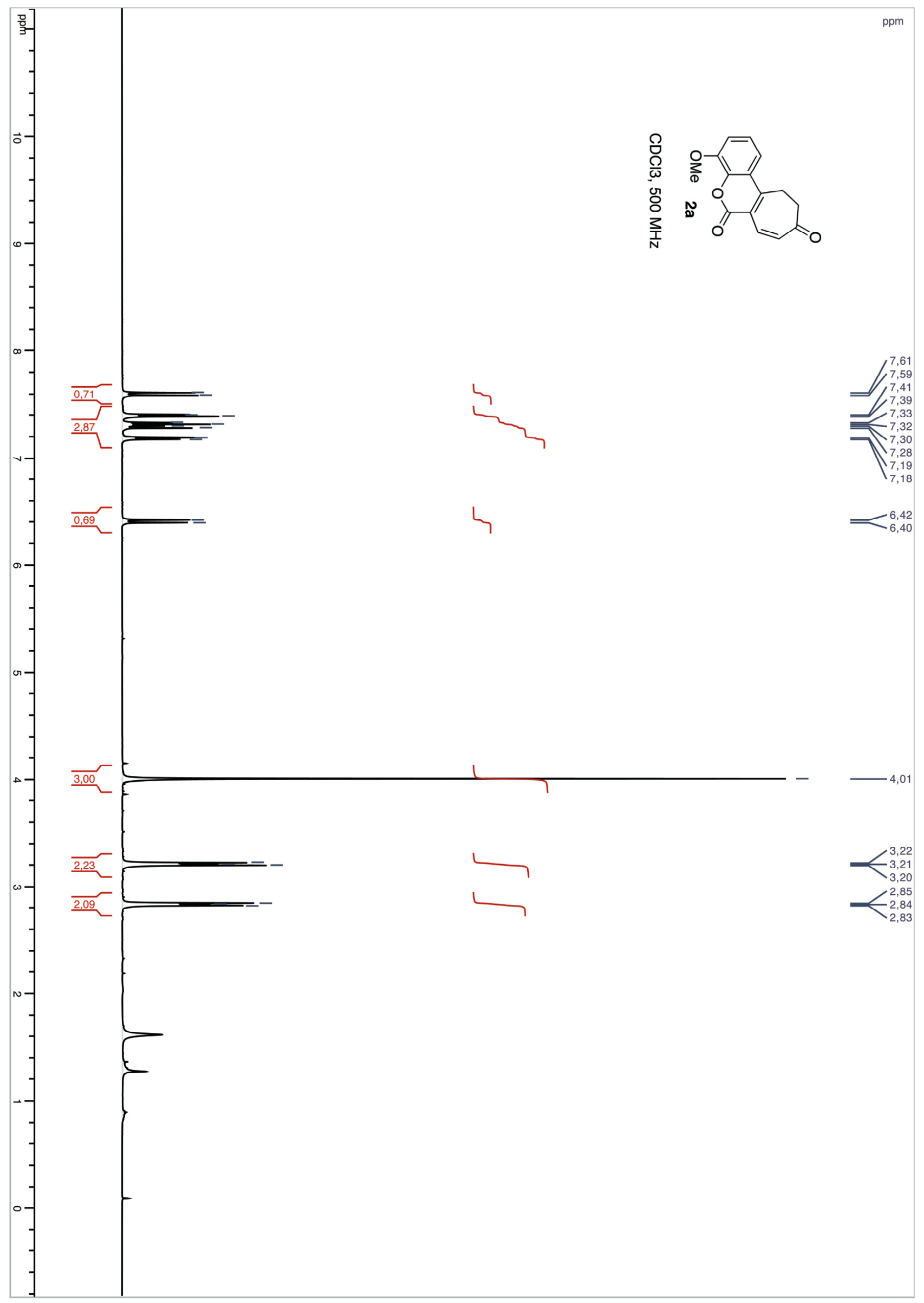




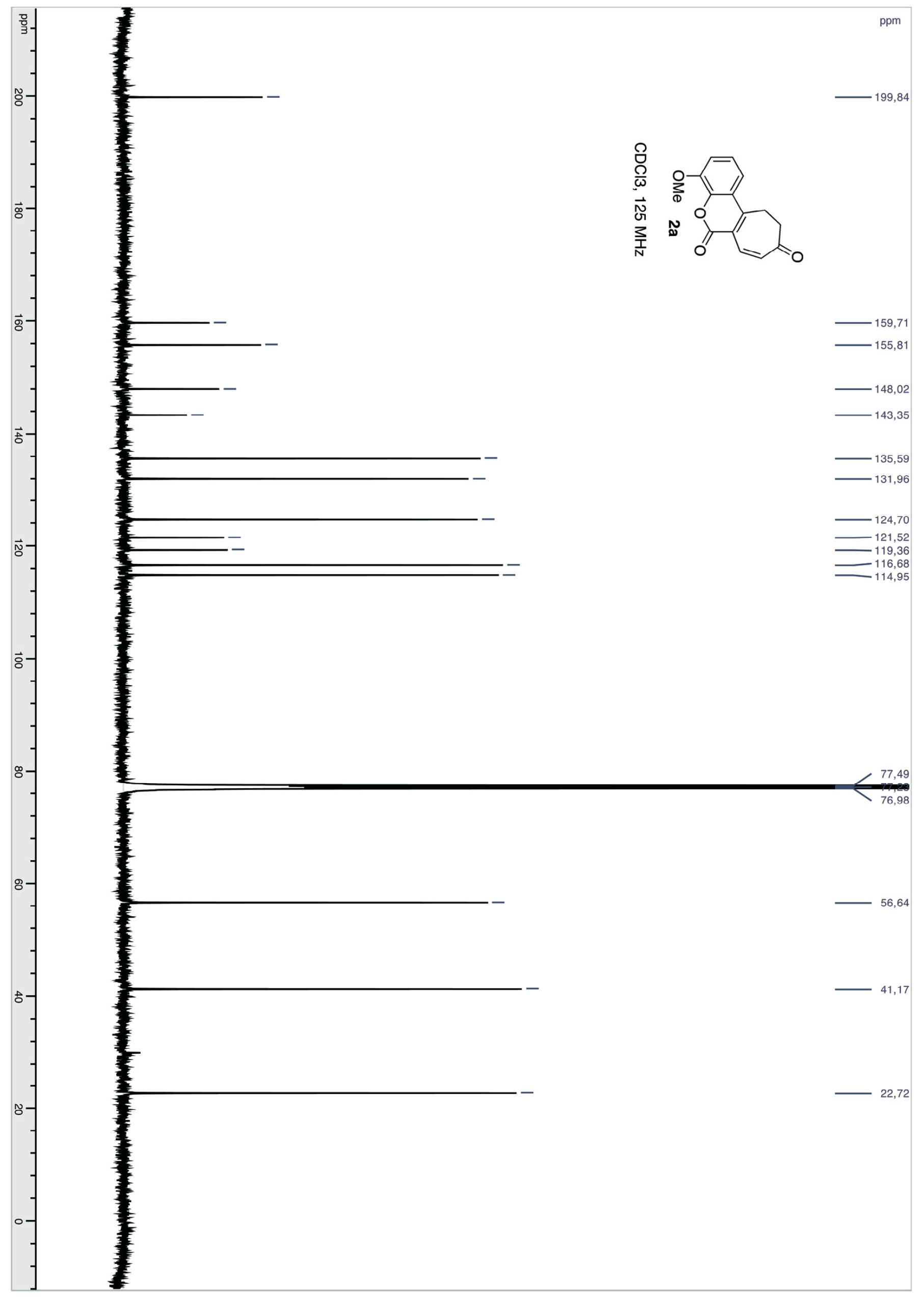




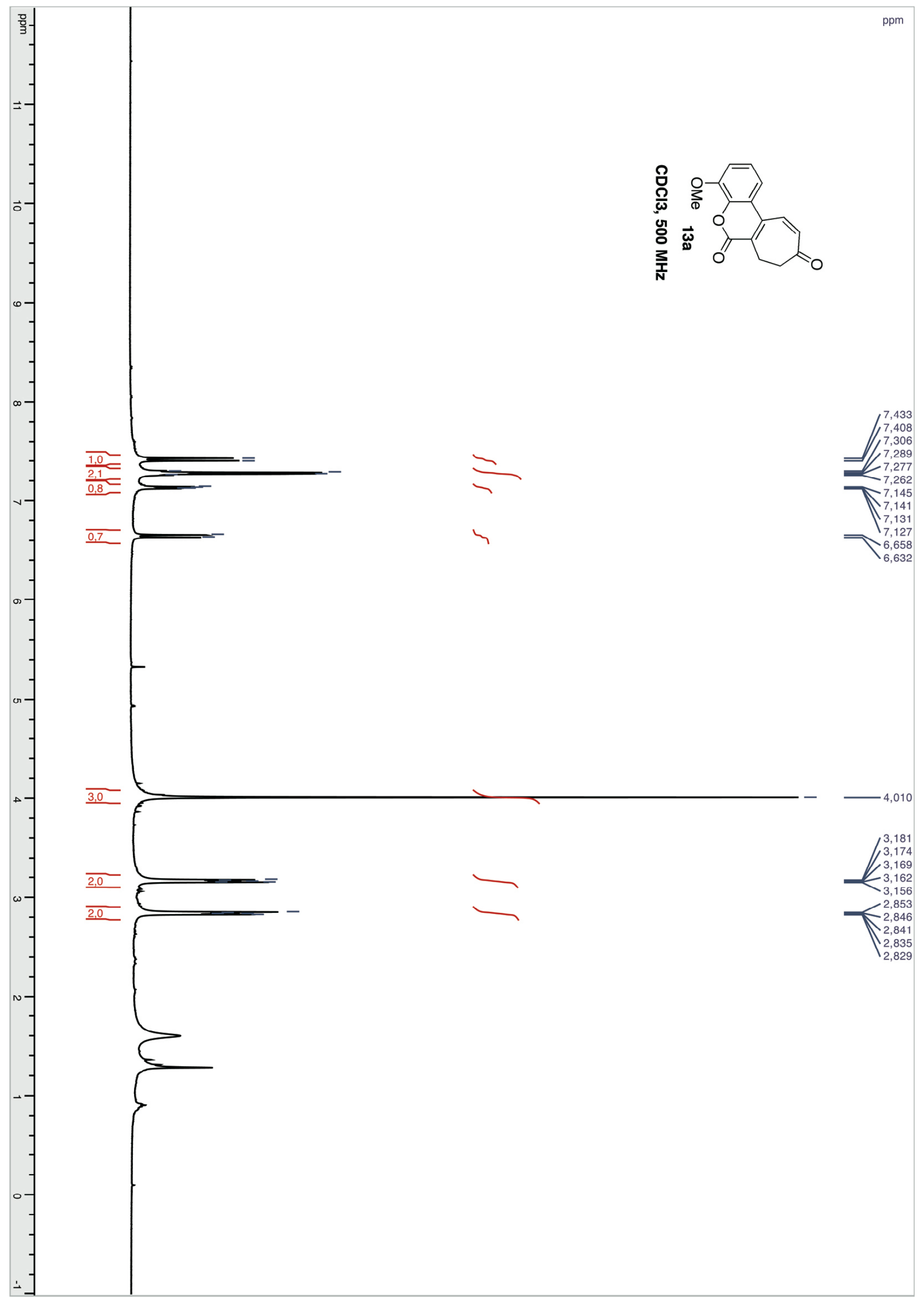




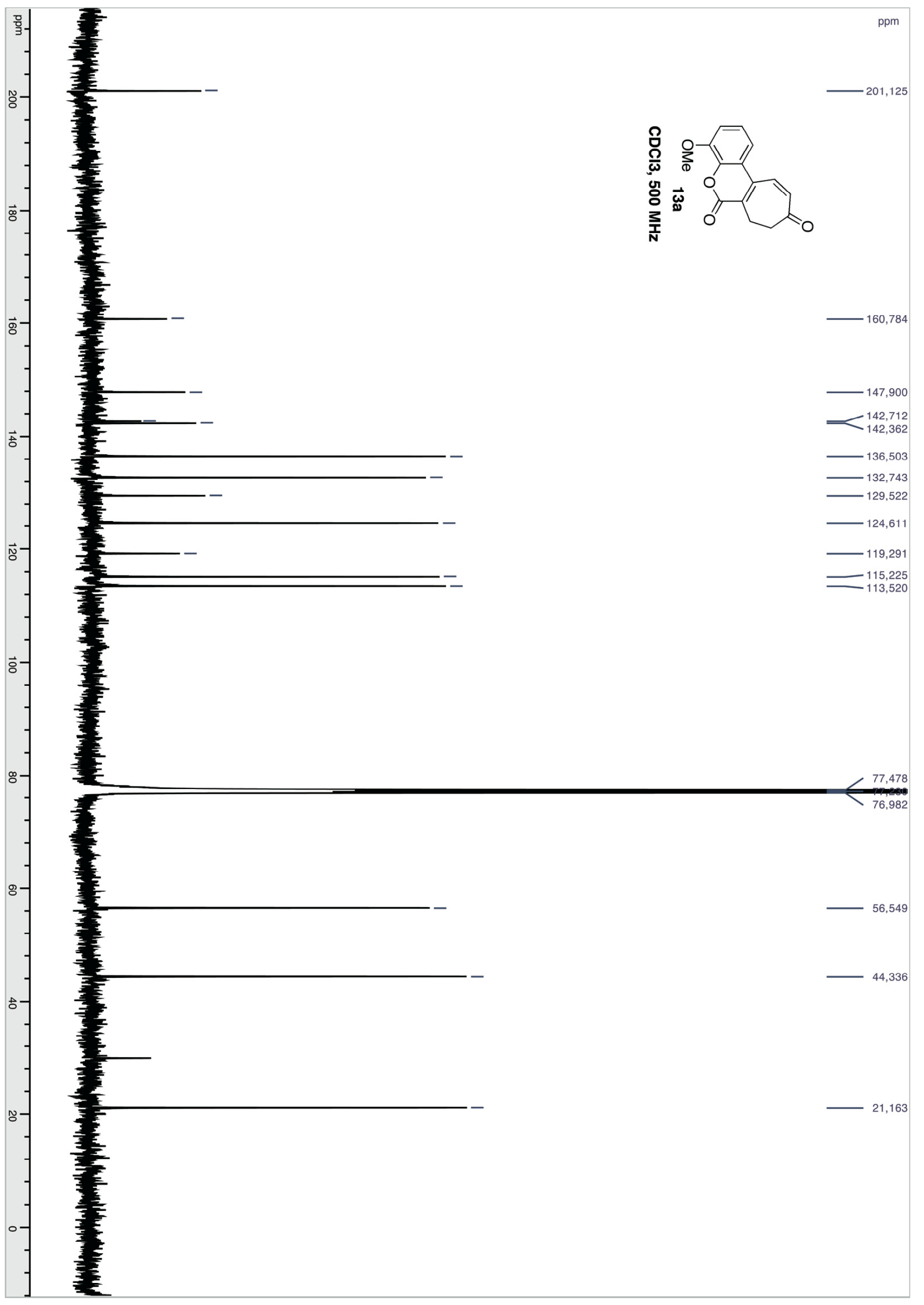

S7 


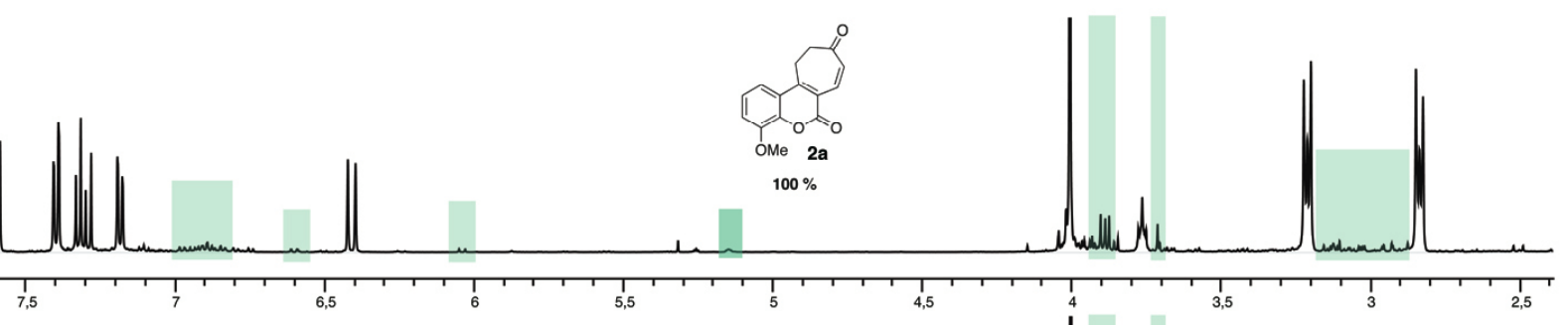

$6 \min$

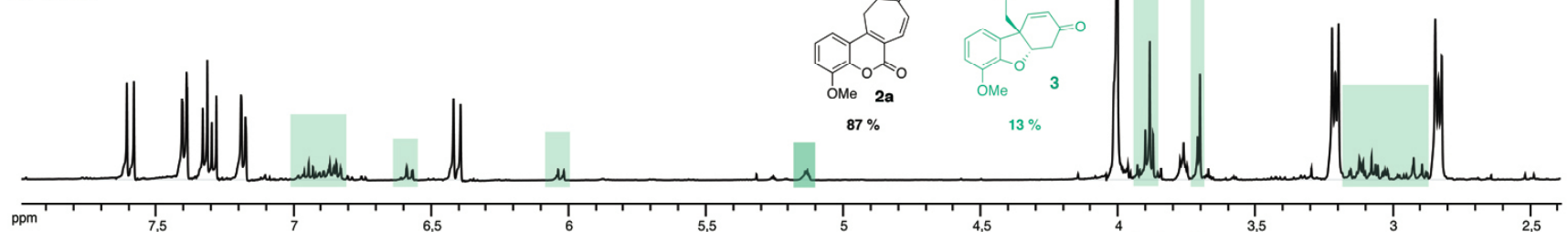

$3 \min$
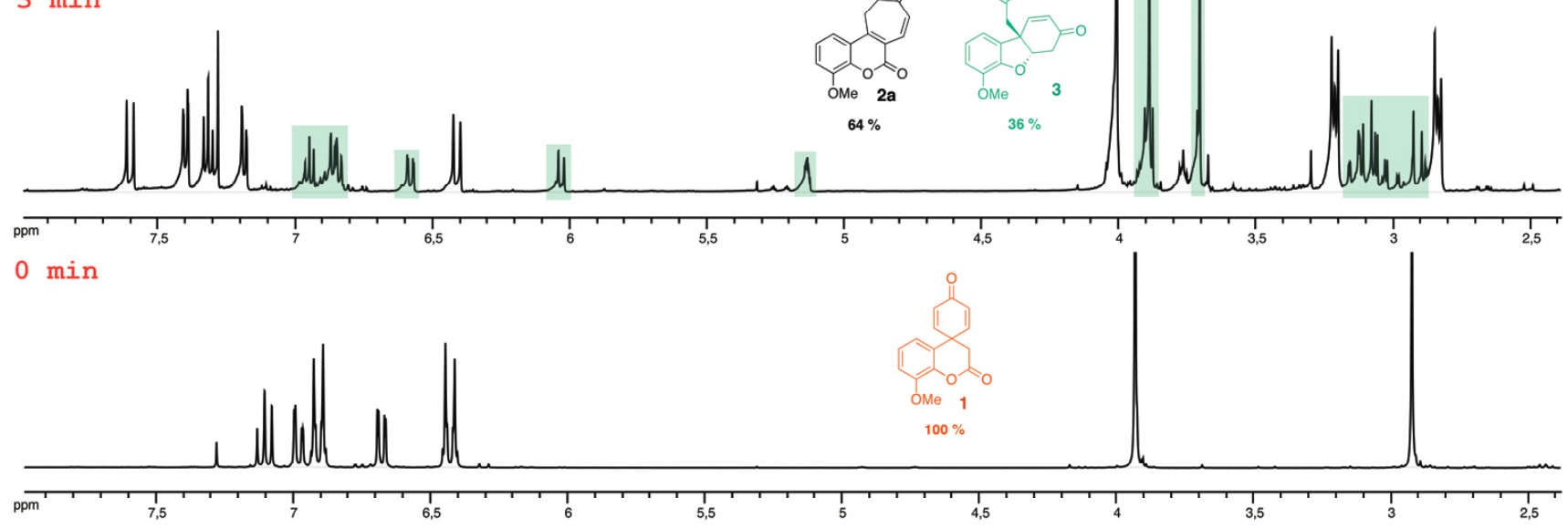

Figure S1. Superposed ${ }^{1} \mathrm{H}-\mathrm{NMR}$ spectra $\left(500 \mathrm{MHz}, \mathrm{CDCl}_{3}\right.$ ) of reaction mixture (from the reaction of 1 with $\mathrm{MeONa}$ in $\mathrm{MeOH}$ at $40^{\circ} \mathrm{C}$ ) after acidic quench at different times. The competitive formation of $\mathbf{2} \mathbf{a}$ and $\mathbf{3}$ (green) is observed, then the conversion of $\mathbf{3}$ in $\mathbf{2 a}$. The rearrangement product $\mathbf{2 a}$ is obtained almost exclusively after $30 \mathrm{~min}$. 


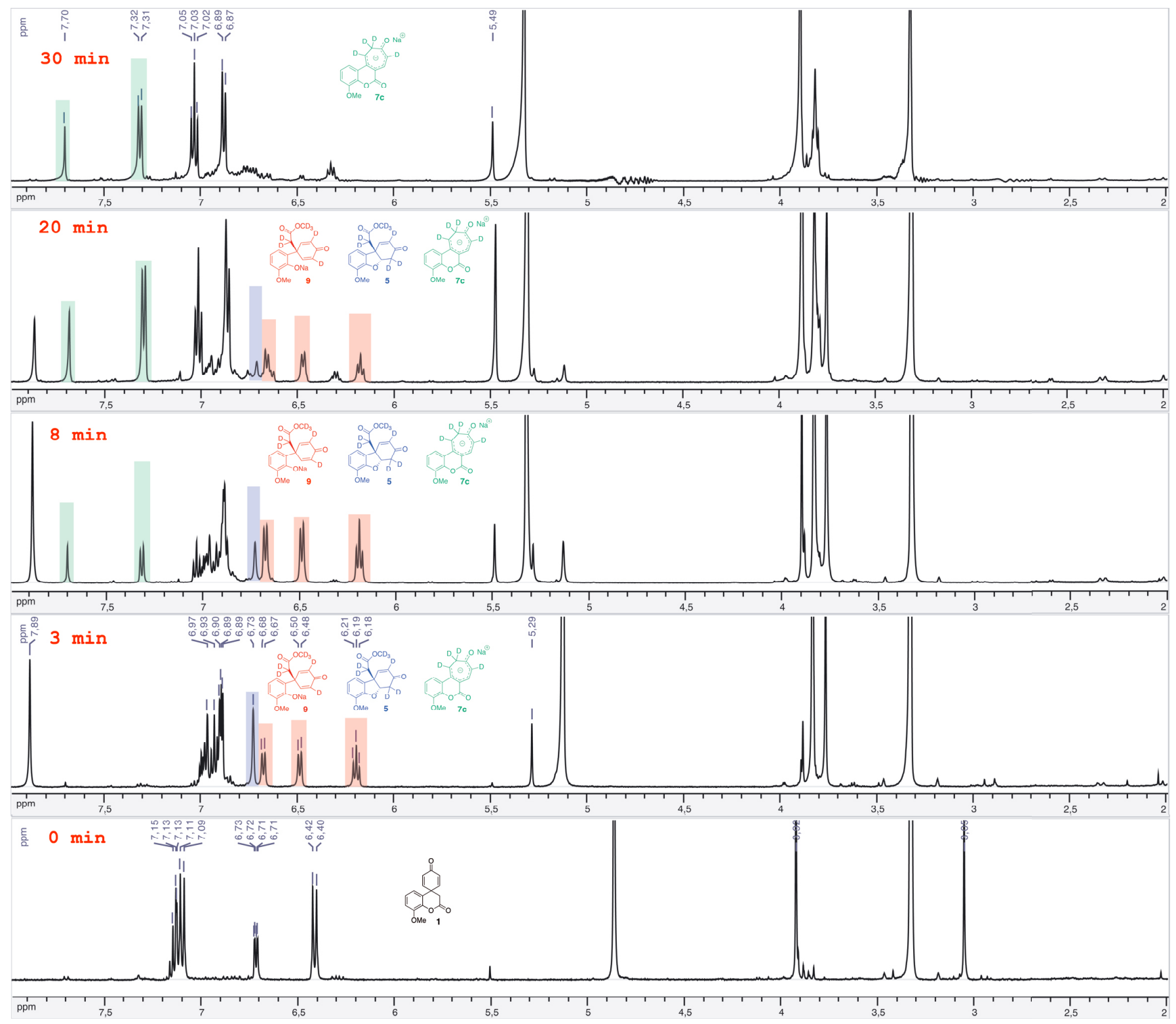

Figure S2. Superposed ${ }^{1} \mathrm{H}-\mathrm{NMR}$ spectra $\left(500 \mathrm{MHz}, \mathrm{CD}_{3} \mathrm{OD}\right)$ of intermediates from the reaction of $\mathbf{1}$ with $\mathrm{CD}_{3} \mathrm{ONa}$ in $\mathrm{CD}_{3} \mathrm{OD}$ at $40^{\circ} \mathrm{C}$. The competitive formation of the ester $\mathbf{5}$, phenoxide $\mathbf{9}$ and 7 -membered ring anion $7 \mathrm{c}$ is observed. The equilibrium is shifted almost completely towards the more thermodynamically stable anion $7 \mathrm{c}$ after $30 \mathrm{~min}$. 


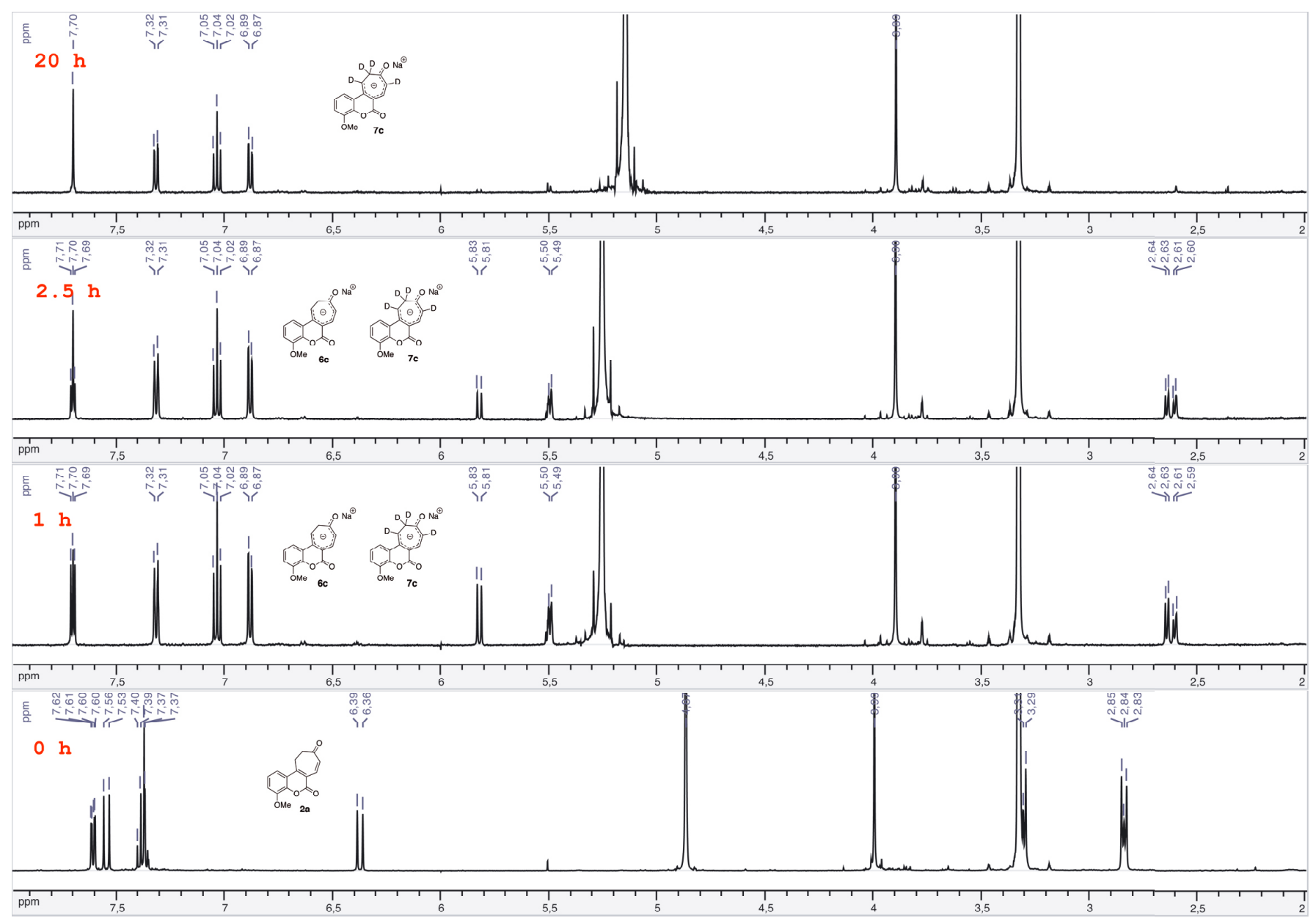

Figure S3. Superposed ${ }^{1} \mathrm{H}-\mathrm{NMR}$ spectra $\left(500 \mathrm{MHz}, \mathrm{CD}_{3} \mathrm{OD}\right)$ of intermediates from the reaction of $2 \mathbf{a}$ with $\mathrm{CD}_{3} \mathrm{ONa}$ in $\mathrm{CD}_{3} \mathrm{OD}$ at room temperature. We observe the immediate formation of the anion $6 \mathrm{c}$, which is progressively deuterated, leading to the complete formation of the anion $7 \mathrm{c}$ after $20 \mathrm{~h}$. 


\section{X-RAY STRUCTURE ANALYSIS OF COMPOUND 2A}

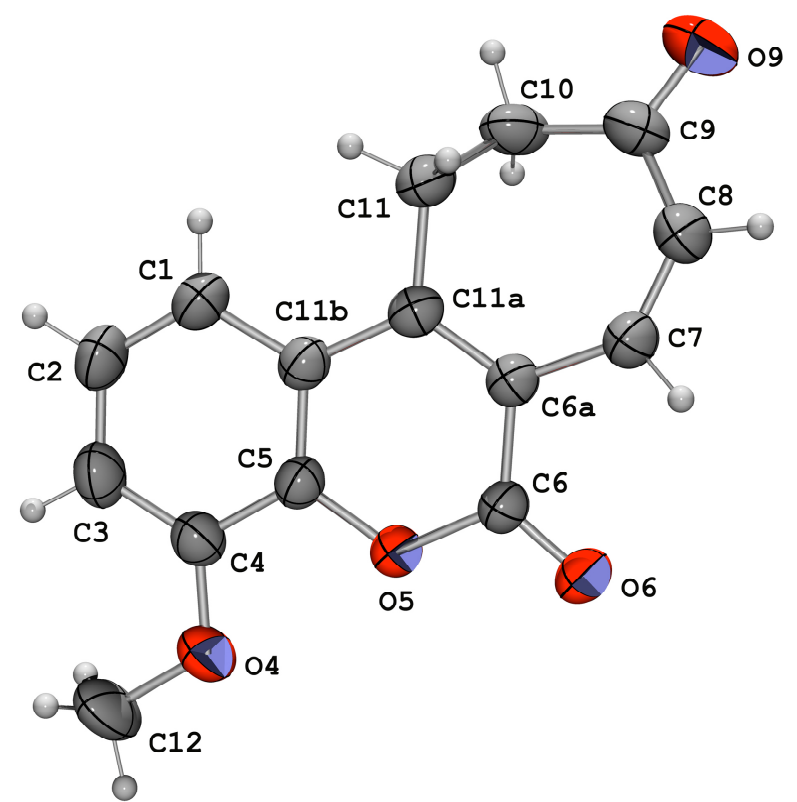

Figure S4. ORTEP drawing of the X-ray structure of 2a shown as displacement ellipsoids at the $30 \%$ probability level
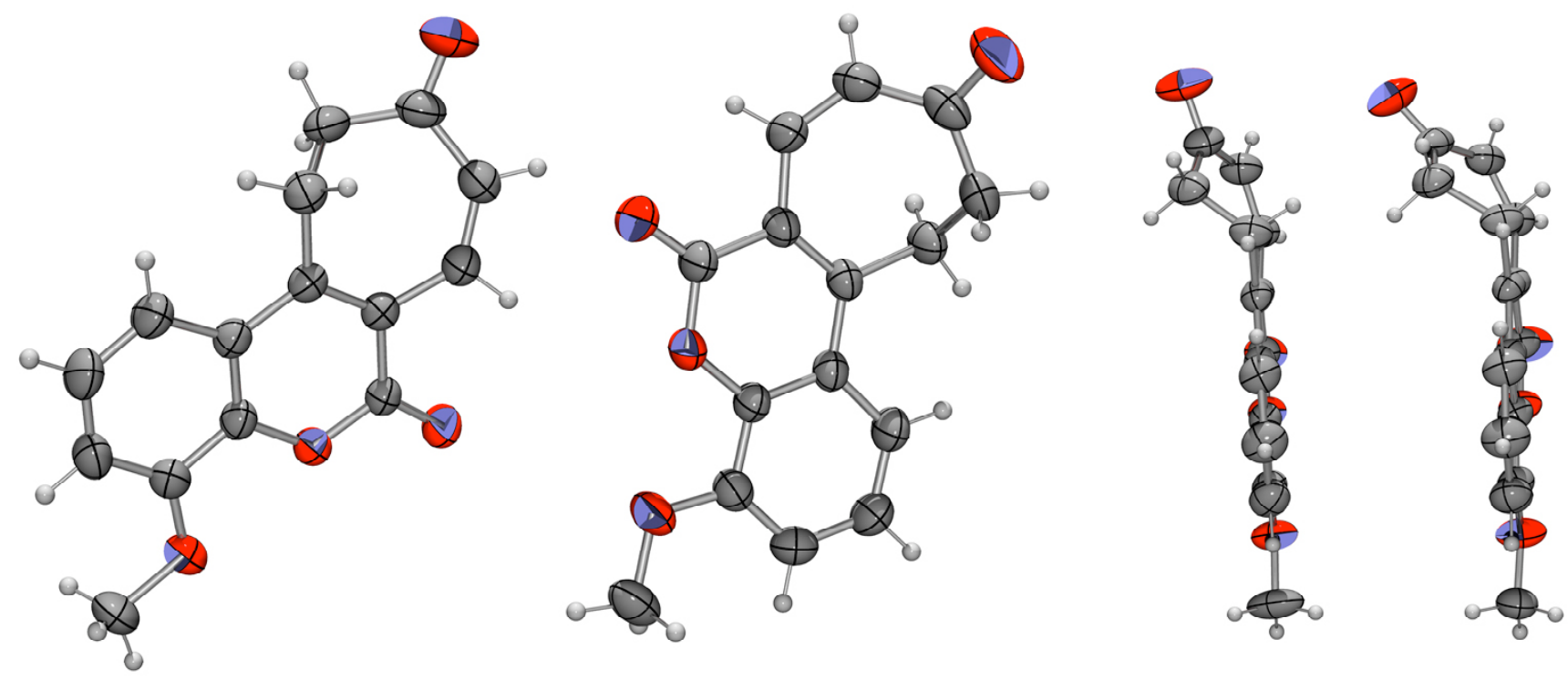

Figure S5. ORTEP drawing of the asymmetric unit (left) and deviations of the atoms C8, C9, C10 and O9 from the molecule plane for the two molecules in the asymmetric unit. Displacement ellipsoids are shown at the $30 \%$ probability level.

Crystals suitable for an X-ray study have been obtained for the 6,7-dihydrotropone 2a and the tridimensional structure is shown in Figure S4. All atoms are coplanar except the atoms C8, C9, C10 and O9. There are two independent molecules in the asymmetric unit, the main difference between them being the degree of deviation of the atoms C8, C9, C10 and O9 from the molecule plane (Figure S5). 
Crystallographic results. Data were obtained from a small colorless crystal plate $(0.55 \times 0.50 \times 0.20 \mathrm{~mm})$. Empirical formula : $\mathrm{C}_{15} \mathrm{H}_{12} \mathrm{O}_{4}, \mathrm{M}_{\mathrm{w}}=256.25$. The compound crystallizes in the triclinic system, space group P-1, racemic. There are four molecules in the unit-cell $(\mathrm{Z}=4)$ and so, two independent molecules in the asymmetric unit. Cell parameters : $a=7.510(5), b=12.824(5), c=13.261(7) \AA, \alpha=73.61(3), \beta=$

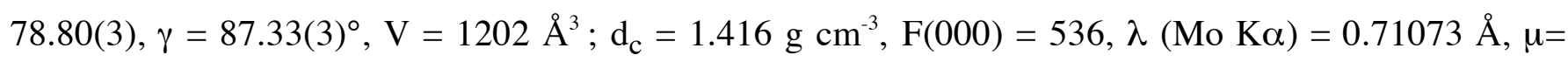
$0.103 \mathrm{~mm}^{-1}$. Data were measured with a Nonius Kappa-CCD linear area-detector diffractometer, using graphite monochromated Mo K $\alpha$ radiation, according to the phi and omega scan method, up to $\theta=$ $27.50^{\circ}$. A total of 9306 intensity data was collected reduced to 7000 triclinic reflections of which 5273 were unique $(\mathrm{Rint}=0.020) .{ }^{1}$ Structure was solved with program $S H E L X S 86^{2}$ and refined by full-matrix least-squares, upon all unique $\mathrm{F}^{2}$ with program SHELXL97. ${ }^{3}$ All the hydrogen atoms were located in difference Fourier maps. They were fitted at theoretical positions and treated as riding, assigned of an isotropic displacement parameter equivalent to 1.12 the one of the bonded atom, 1.15 for those of the methyl groups. Refinement of 345 parameters converged to $\mathrm{R} 1(\mathrm{~F})=0.0486$ with the 3639 observed reflections having $\mathrm{I} \geq 2$ sigma $(\mathrm{I})$ and $\mathrm{wR} 2\left(\mathrm{~F}^{2}\right)=0.1272$ with all the 5273 unique data; goodness-of-fit $\mathrm{S}$ factor of 1.024. The residual electron density was found between -0.18 and $0.19 \mathrm{e}^{-3}$. All the crystallographic results are given in Cif file and Tables S1 to S6.

Comparison of molecular conformations. The two independent molecules of the asymmetric unit named respectively, * and **, appear in Figure S5, showing the same diastereoisomer. In both molecules, bond distance and angle values are nearly the same with double bonds C6a-C11a (1.362 A) and C7-C8 (av. $1.341 \AA$ ) clearly precised. However, differences appear in torsion angle values of the seven membered rings principally along the bonds: C8-C9 (-6.1 in *, $-28.1^{\circ}$ in **), C9-C10 (-43.7 and $\left.-19.0^{\circ}\right)$ and $\mathrm{C} 10$ $\mathrm{C} 11$ (76.9 and 86.2), indicating differences in the ring conformations. Fitting the two structures, atoms C7 and C11 are quitely coplanar with the bicyclic aromatic part in each molecule, while atoms C8, C9 and C10 are respectively deviated by $0.226(2), 0.885(2)$ and $1.183(2) \AA$ in *, by $0.500(2), 1.372(2)$ and 1.284(2) $\AA$ in **, from the mean plane of the other twelve atoms. If atom C8 is included in the aromatic mean plane, deviations of atoms C9 and C10 become : 0.800(2) and 1.114(2) $\AA$ in *,1.180(2) and 1.144 (2) $\AA$ - nearly the same - in **. So, the angles between the planes [C11, C11a, C6a, C7, C8] and [C8, C9, $\mathrm{C} 10, \mathrm{C} 11]$ in the seven membered rings, are respectively $136.3^{\circ}$ in $*, 131.0^{\circ}$ in $* *$. More, if we consider only the mean plane of the double bonds involving atoms [C11a, C6a, C7, C8, C9], atoms C10 and C11 are deviated from this plane by respectively $0.327(2)$ and $-0.620(2) \AA$ in *, while atom $\mathrm{C} 10$ lies in this plane in **, with C11 situated below by $-0.890(2) \AA$. The crystal packing study shows that only van der Waals contacts exist between the molecules.

\footnotetext{
${ }^{1}$ Collect (Nonius BV, 1997-2000); HKL Denzo and Scalepack (Otinowski \& Minor, 1997).

${ }^{2}$ Sheldrick, G.M. (1990). SHELXS86. Acta Cryst. A 46, 467-473.

${ }^{3}$ Sheldrick, G.M. (1997). SHELX97. Program for the Refinement of Crystal Structures, Univ. of Göttingen, Germany.
} 
Table s1. Crystal data and structure refinement for compound $\mathbf{2 a}$.

\begin{tabular}{|c|c|}
\hline Identification code & 'VARIN051' \\
\hline Empirical formula & C15 H12 O4 \\
\hline Formula weight & 256.25 \\
\hline Temperature & $293(2) \mathrm{K}$ \\
\hline Wavelength & $0.71073 \mathrm{~A}$ \\
\hline Crystal system, space group & Triclinic, $\quad P-1$ \\
\hline Unit cell dimensions & $\begin{array}{llll}\mathrm{a}=7.510(5) \mathrm{A} & \text { alpha }=73.61(3) \text { deg. } \\
\mathrm{b}=12.824(5) \mathrm{A} & \text { beta }=78.80(3) \text { deg. } \\
\mathrm{c}=13.261(7) \mathrm{A} & \text { gamma }=87.33(3) \text { deg. }\end{array}$ \\
\hline Volume & $1201.9(11) A^{\wedge} 3$ \\
\hline z, Z', Calculated density & $4,2,1.416 \mathrm{Mg} / \mathrm{m}^{\wedge} 3$ \\
\hline Absorption coefficient & $0.103 \mathrm{~mm}^{\wedge}-1$ \\
\hline$F(000)$ & 536 \\
\hline Crystal size & $0.55 \times 0.50 \times 0.20 \mathrm{~mm}$ \\
\hline Theta range for data collection & 2.76 to $27.50 \mathrm{deg}$. \\
\hline Limiting indices & $-9<=\mathrm{h}<=9, \quad-16<=\mathrm{k}<=16, \quad-16<=1<=17$ \\
\hline Reflections collected / unique & $9306-7000 / 5273[R($ int $)=0.0199]$ \\
\hline Completeness to theta $=27.50$ & $95.3 \div$ \\
\hline Absorption correction & Not measured \\
\hline Refinement method & Full-matrix least-squares on $\mathrm{F}^{\wedge} 2$ \\
\hline Data / restraints / parameters & $5273 / 0 / 345$ \\
\hline Goodness-of-fit on $F^{\wedge} 2$ & 1.024 \\
\hline Final $R$ indices [I>2sigma(I)] & $\mathrm{R} 1=0.0486, \mathrm{WR} 2=0.1101(3639 \mathrm{Fo})$ \\
\hline $\mathrm{R}$ indices (all data) & $\mathrm{R} 1=0.0779, \mathrm{wR} 2=0.1272(5273$ data $)$ \\
\hline Largest diff. peak and hole & 0.187 and -0.177 e. $A^{\wedge}-3$ \\
\hline
\end{tabular}


Table s2. Atomic coordinates $\left(x 10^{\wedge} 4\right)$ and equivalent isotropic displacement parameters $\left(A^{\wedge} 2 \times 10^{\wedge} 3\right)$ for compound $2 a$.

$\mathrm{U}(\mathrm{eq})$ is defined as one third of the trace of the orthogonalized Uij tensor. (* Molecule 1, ** Molecule 2)

\begin{tabular}{|c|c|c|c|c|}
\hline & $\mathrm{x}$ & $\mathrm{y}$ & $\mathrm{z}$ & $\mathrm{U}(\mathrm{eq})$ \\
\hline * $\mathrm{C} 1$ & $5205(3)$ & $3658(2)$ & $2140(2)$ & $54(1)$ \\
\hline $\mathrm{C} 2$ & $4806(3)$ & $2587(2)$ & $2304(2)$ & $62(1)$ \\
\hline C3 & $5442(3)$ & $2054(2)$ & $1534(2)$ & $57(1)$ \\
\hline $\mathrm{C} 4$ & $6506(3)$ & $2600(1)$ & $571(1)$ & $48(1)$ \\
\hline C5 & $6902(2)$ & $3696(1)$ & $402(1)$ & $41(1)$ \\
\hline 05 & $7928(2)$ & $4211(1)$ & $-580(1)$ & $46(1)$ \\
\hline C6 & $8399(2)$ & $5287(1)$ & $-854(1)$ & $41(1)$ \\
\hline $\mathrm{c} 6 \mathrm{a}$ & $7777(2)$ & $5888(1)$ & $-64(1)$ & $40(1)$ \\
\hline C7 & $8222(3)$ & $7045(1)$ & $-455(1)$ & 49 (1) \\
\hline $\mathrm{C} 8$ & $8325(3)$ & $7798(2)$ & $62(2)$ & $58(1)$ \\
\hline C9 & $8167(3)$ & $7658(2)$ & $1207(2)$ & $58(1)$ \\
\hline $\mathrm{C} 10$ & $7663(3)$ & $6568(2)$ & $1970(2)$ & $59(1)$ \\
\hline $\mathrm{C} 11$ & $6121(3)$ & $6017(2)$ & $1708(1)$ & $54(1)$ \\
\hline C11a & $6766(2)$ & $5385(1)$ & $910(1)$ & $41(1)$ \\
\hline $\mathrm{C} 11 \mathrm{~b}$ & $6285(2)$ & $4246(1)$ & $1170(1)$ & $42(1)$ \\
\hline $\mathrm{C} 12$ & $6884(4)$ & $1034(2)$ & $-73(2)$ & $85(1)$ \\
\hline 04 & $7196(2)$ & $2168(1)$ & $-249(1)$ & $62(1)$ \\
\hline 06 & $9313(2)$ & $5647(1)$ & $-1733(1)$ & $56(1)$ \\
\hline 09 & $8478(3)$ & $8426(1)$ & $1518(1)$ & $90(1)$ \\
\hline ** C1 & $9034(3)$ & $1683(2)$ & $3263(1)$ & $53(1)$ \\
\hline C2 & $8869(3)$ & $922(2)$ & $2744(2)$ & $60(1)$ \\
\hline C3 & $8165(3)$ & $-106(2)$ & $3296(2)$ & $56(1)$ \\
\hline $\mathrm{C} 4$ & $7624(3)$ & $-378(1)$ & $4389(1)$ & $47(1)$ \\
\hline C5 & $7810(2)$ & $394(1)$ & $4922(1)$ & $42(1)$ \\
\hline 05 & $7255(2)$ & $74(1)$ & $6015(1)$ & $50(1)$ \\
\hline $\mathrm{C} 6$ & $7373(3)$ & $757(1)$ & $6629(1)$ & $48(1)$ \\
\hline $\mathrm{c} 6 \mathrm{a}$ & $8072(2)$ & $1858(1)$ & $6080(1)$ & $42(1)$ \\
\hline C7 & $8174(3)$ & $2531(1)$ & $6792(1)$ & $49(1)$ \\
\hline $\mathrm{C} 8$ & $8020(3)$ & $3609(2)$ & $6606(2)$ & $53(1)$ \\
\hline C9 & 7519 (3) & $4385(1)$ & $5663(2)$ & $53(1)$ \\
\hline $\mathrm{C} 10$ & $7904(3)$ & $4170(1)$ & $4592(2)$ & $58(1)$ \\
\hline $\mathrm{C} 11$ & $9339(3)$ & $3317(1)$ & $4460(1)$ & $50(1)$ \\
\hline $\mathrm{C} 11 \mathrm{a}$ & $8623(2)$ & $2182(1)$ & $4997(1)$ & $40(1)$ \\
\hline $\mathrm{C} 11 \mathrm{~b}$ & $8504(2)$ & $1432(1)$ & $4377(1)$ & $41(1)$ \\
\hline $\mathrm{C} 12$ & $6606(3)$ & $-2132(2)$ & $4486(2)$ & $66(1)$ \\
\hline 04 & $6921(2)$ & $-1359(1)$ & $5015(1)$ & $64(1)$ \\
\hline 06 & $6862(2)$ & $398(1)$ & $7580(1)$ & $72(1)$ \\
\hline 09 & $6781(2)$ & $5233(1)$ & $5757(1)$ & $83(1)$ \\
\hline
\end{tabular}


Table s5. Bond lengths [A] and angles [deg] for compound 2a.

\begin{tabular}{|c|c|}
\hline $\mathrm{C} 1-\mathrm{C} 2$ & \\
\hline $\mathrm{C} 1-\mathrm{C} 2$ & $\begin{array}{l}1.367(3) \\
1.409(3)\end{array}$ \\
\hline $\mathrm{C} 1-\mathrm{C} 11 \mathrm{~b}$ & $1.409(3)$ \\
\hline $\mathrm{C} 2-\mathrm{C} 3$ & $1.383(3)$ \\
\hline $\mathrm{C} 3-\mathrm{C} 4$ & $1.382(3)$ \\
\hline $\mathrm{C} 4-04$ & $1.361(2)$ \\
\hline $\mathrm{C} 4-\mathrm{C} 5$ & $1.397(2)$ \\
\hline C5-O5 & $1.378(2)$ \\
\hline $\mathrm{C} 5-\mathrm{C} 11 \mathrm{~b}$ & $1.393(2)$ \\
\hline $\mathrm{O} 5-\mathrm{C} 6$ & $1.369(2)$ \\
\hline C6-06 & $1.207(2)$ \\
\hline c6-c6a & $1.464(2)$ \\
\hline C6a-C11a & $1.362(2)$ \\
\hline $\mathrm{C} 6 \mathrm{a}-\mathrm{C} 7$ & $1.458(2)$ \\
\hline C7-C 8 & $1.345(2)$ \\
\hline $\mathrm{C} 8-\mathrm{C} 9$ & $1.460(3)$ \\
\hline C9-09 & $1.218(2)$ \\
\hline $\mathrm{C} 9-\mathrm{C} 10$ & $1.491(3)$ \\
\hline $\mathrm{C} 10-\mathrm{C} 11$ & $1.526(3)$ \\
\hline C11-C11a & $1.504(2)$ \\
\hline $\mathrm{C} 11 \mathrm{a}-\mathrm{C} 11 \mathrm{~b}$ & $1.449(2)$ \\
\hline C12-04 & $1.429(2)$ \\
\hline * $\mathrm{C} 2-\mathrm{C} 1-\mathrm{C} 11 \mathrm{~b}$ & $120.08(18)$ \\
\hline $\mathrm{C} 1-\mathrm{C} 2-\mathrm{C} 3$ & $121.49(18)$ \\
\hline $\mathrm{C} 4-\mathrm{C} 3-\mathrm{C} 2$ & $120.31(17)$ \\
\hline $\mathrm{O} 4-\mathrm{C} 4-\mathrm{C} 3$ & $125.72(16)$ \\
\hline $\mathrm{O} 4-\mathrm{C} 4-\mathrm{C} 5$ & $116.13(16)$ \\
\hline C $3-C 4-C 51$ & $118.15(16)$ \\
\hline O5-C5-C11b & $121.67(15)$ \\
\hline $\mathrm{O} 5-\mathrm{C} 5-\mathrm{C} 4$ & $115.90(15)$ \\
\hline $\mathrm{C} 11 \mathrm{~b}-\mathrm{C} 5-\mathrm{C} 4$ & $122.42(16)$ \\
\hline $\mathrm{C} 6-05-\mathrm{C} 5$ & $121.93(13)$ \\
\hline $06-C 6-05$ & $116.09(14)$ \\
\hline $06-C 6-C 6 a$ & $126.08(15)$ \\
\hline $05-\mathrm{C} 6-\mathrm{C} 6 \mathrm{a}$ & $117.83(14)$ \\
\hline $\mathrm{C} 11 \mathrm{a}-\mathrm{C} 6 \mathrm{a}-\mathrm{C} 7$ & $125.30(15)$ \\
\hline $\mathrm{C} 11 \mathrm{a}-\mathrm{C} 6 \mathrm{a}-\mathrm{C} 6$ & $120.60(15)$ \\
\hline $\mathrm{C} 7-\mathrm{C} 6 \mathrm{a}-\mathrm{C} 6$ & $113.93(15)$ \\
\hline $\mathrm{C} 8-\mathrm{C} 7-\mathrm{C} 6 \mathrm{a}$ & $131.71(17)$ \\
\hline C7-C8-C9 & $129.12(18)$ \\
\hline O9-C9-C8 & $119.4(2)$ \\
\hline O9-C $9-\mathrm{C} 10$ & $121.46(19)$ \\
\hline $\mathrm{C} 8-\mathrm{C} 9-\mathrm{C} 10$ & $119.08(16)$ \\
\hline $\mathrm{C} 9-\mathrm{C} 10-\mathrm{C} 11$ & $113.17(17)$ \\
\hline $\mathrm{C} 11 \mathrm{a}-\mathrm{C} 11-\mathrm{C} 10$ & $113.11(16)$ \\
\hline $\mathrm{C} 6 \mathrm{a}-\mathrm{C} 11 \mathrm{a}-\mathrm{C} 11 \mathrm{~b}$ & $120.06(15)$ \\
\hline $\mathrm{C} 6 \mathrm{a}-\mathrm{C} 11 \mathrm{a}-\mathrm{C} 11$ & $119.97(15)$ \\
\hline $\mathrm{C} 11 \mathrm{~b}-\mathrm{C} 11 \mathrm{a}-\mathrm{C} 11$ & $119.96(15)$ \\
\hline $\mathrm{C} 5-\mathrm{C} 11 \mathrm{~b}-\mathrm{C} 1$ & $117.54(16)$ \\
\hline $\mathrm{C} 5-\mathrm{C} 11 \mathrm{~b}-\mathrm{C} 11 \mathrm{a}$ & $117.90(15)$ \\
\hline $\mathrm{C} 1-\mathrm{C} 11 \mathrm{~b}-\mathrm{C} 11 \mathrm{a}$ & $124.55(16)$ \\
\hline $\mathrm{C} 4-\mathrm{O} 4-\mathrm{C} 12$ & $117.01(16)$ \\
\hline
\end{tabular}




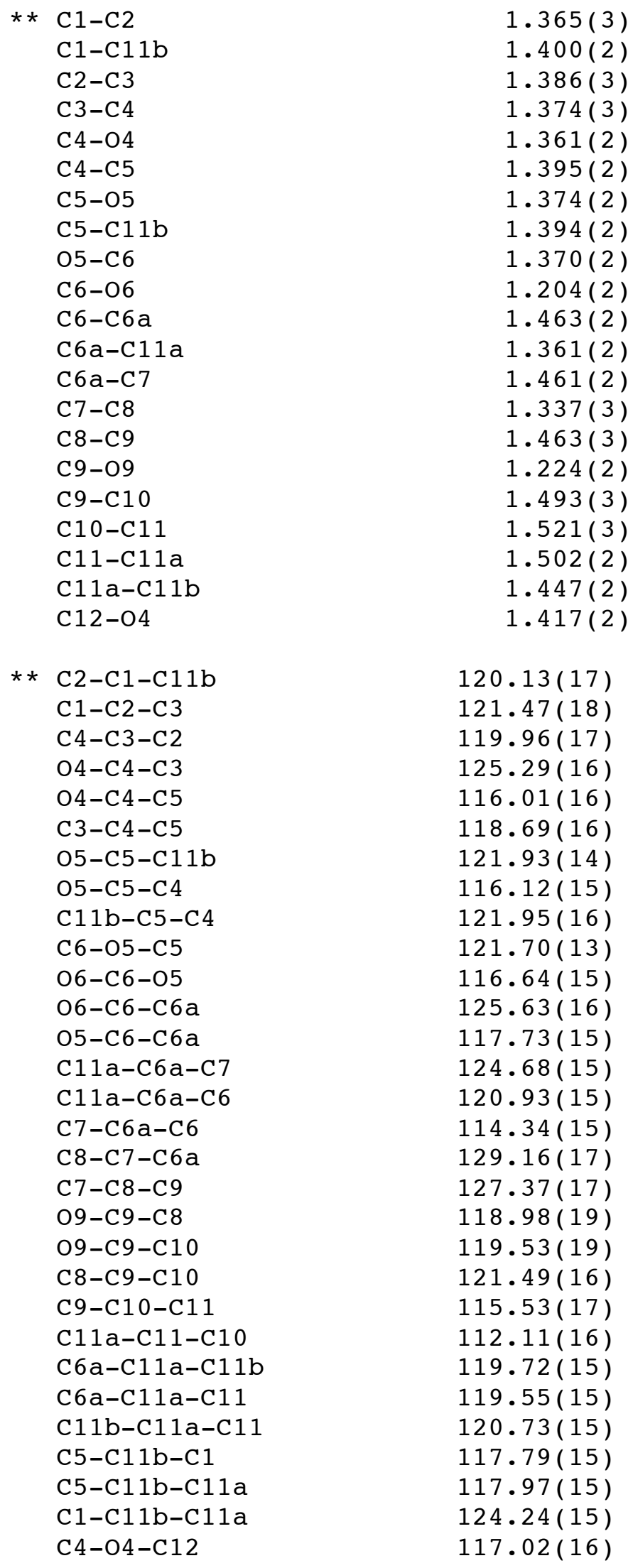


Table S6. Anisotropic displacement parameters $\left(A^{\wedge} 2 \times 10^{\wedge} 3\right)$ for compound $\mathbf{2 a}$.

The anisotropic displacement factor exponent takes the form: $-2 \mathrm{pi}^{\wedge} 2\left[\mathrm{~h} 2 \mathrm{a}{ }^{\wedge} 2 \mathrm{U} 11+\ldots+2 \mathrm{~h} \mathrm{k} a * b * \mathrm{U} 12\right.$ ]

\begin{tabular}{|c|c|c|c|c|c|c|}
\hline & & $\mathrm{U} 22$ & U33 & $\mathrm{U} 23$ & $\mathrm{U} 13$ & $\mathrm{U} 12$ \\
\hline $\mathrm{C} 1$ & $59(1)$ & $57(1)$ & $41(1)$ & $-11(1)$ & $0(1)$ & $-9(1)$ \\
\hline $\mathrm{C} 2$ & $71(1)$ & $58(1)$ & $46(1)$ & $-2(1)$ & $1(1)$ & $-18(1)$ \\
\hline C3 & $68(1)$ & $44(1)$ & $53(1)$ & $-3(1)$ & $-12(1)$ & $-13(1)$ \\
\hline $\mathrm{C} 4$ & $57(1)$ & $43(1)$ & $44(1)$ & $-12(1)$ & $-13(1)$ & $-5(1)$ \\
\hline C5 & $43(1)$ & $42(1)$ & $35(1)$ & $-7(1)$ & $-5(1)$ & $-5(1)$ \\
\hline 05 & $56(1)$ & $40(1)$ & $38(1)$ & $-12(1)$ & $0(1)$ & $-5(1)$ \\
\hline $\mathrm{C} 6$ & $46(1)$ & $40(1)$ & $34(1)$ & $-7(1)$ & $-4(1)$ & $-2(1)$ \\
\hline $\mathrm{C} 6 \mathrm{a}$ & $43(1)$ & $40(1)$ & $37(1)$ & $-11(1)$ & $-7(1)$ & 0 (1) \\
\hline $\mathrm{C} 7$ & $61(1)$ & $44(1)$ & $39(1)$ & $-9(1)$ & $-3(1)$ & $-2(1)$ \\
\hline C8 & $74(1)$ & $43(1)$ & $54(1)$ & $-15(1)$ & $-3(1)$ & $-5(1)$ \\
\hline $\mathrm{C} 9$ & $68(1)$ & $54(1)$ & $60(1)$ & $-30(1)$ & $-10(1)$ & $0(1)$ \\
\hline $\mathrm{C} 10$ & $83(2)$ & $56(1)$ & $43(1)$ & $-23(1)$ & $-14(1)$ & $7(1)$ \\
\hline $\mathrm{C} 11$ & $64(1)$ & $50(1)$ & $43(1)$ & $-16(1)$ & $4(1)$ & $0(1)$ \\
\hline $\mathrm{C} 11 \mathrm{a}$ & $42(1)$ & $45(1)$ & $36(1)$ & $-12(1)$ & $-7(1)$ & $1(1)$ \\
\hline $\mathrm{C} 11 \mathrm{~b}$ & $43(1)$ & $44(1)$ & $36(1)$ & $-8(1)$ & $-6(1)$ & $-2(1)$ \\
\hline C12 & $145(2)$ & $44(1)$ & $68(1)$ & $-21(1)$ & $-15(2)$ & $-18(1)$ \\
\hline 04 & $91(1)$ & $41(1)$ & $54(1)$ & $-17(1)$ & $-7(1)$ & $-10(1)$ \\
\hline 06 & 70 (1) & $48(1)$ & $40(1)$ & $-10(1)$ & $7(1)$ & $-4(1)$ \\
\hline 09 & $134(2)$ & $68(1)$ & $81(1)$ & $-44(1)$ & $-11(1)$ & $-15(1)$ \\
\hline $\mathrm{C} 1$ & $67(1)$ & $46(1)$ & $39(1)$ & $-5(1)$ & $-2(1)$ & $-6(1)$ \\
\hline $\mathrm{C} 2$ & $82(2)$ & $58(1)$ & $38(1)$ & $-12(1)$ & $-5(1)$ & $-6(1)$ \\
\hline C3 & $69(1)$ & $53(1)$ & $52(1)$ & $-23(1)$ & $-12(1)$ & $0(1)$ \\
\hline C4 & $53(1)$ & $40(1)$ & $48(1)$ & $-12(1)$ & $-6(1)$ & $-2(1)$ \\
\hline C5 & $47(1)$ & $38(1)$ & $36(1)$ & $-8(1)$ & $-3(1)$ & $-1(1)$ \\
\hline 05 & $68(1)$ & $38(1)$ & $39(1)$ & $-7(1)$ & $2(1)$ & $-11(1)$ \\
\hline C6 & $58(1)$ & $41(1)$ & $39(1)$ & $-10(1)$ & $1(1)$ & $-6(1)$ \\
\hline $\mathrm{C} 6 \mathrm{a}$ & $44(1)$ & $36(1)$ & $40(1)$ & $-8(1)$ & $-3(1)$ & $-2(1)$ \\
\hline C7 & $57(1)$ & $48(1)$ & $40(1)$ & $-11(1)$ & $-4(1)$ & $-5(1)$ \\
\hline C8 & $57(1)$ & $50(1)$ & $56(1)$ & $-23(1)$ & $-4(1)$ & $-8(1)$ \\
\hline $\mathrm{C} 9$ & $50(1)$ & $37(1)$ & $70(1)$ & $-18(1)$ & $-1(1)$ & $-7(1)$ \\
\hline C10 & $73(1)$ & $36(1)$ & $61(1)$ & $-5(1)$ & $-14(1)$ & $-2(1)$ \\
\hline C11 & $60(1)$ & $39(1)$ & $44(1)$ & $-7(1)$ & $3(1)$ & $-11(1)$ \\
\hline $\mathrm{C} 11 \mathrm{a}$ & $40(1)$ & $35(1)$ & $41(1)$ & $-6(1)$ & $-2(1)$ & $0(1)$ \\
\hline $\mathrm{C} 11 \mathrm{~b}$ & $43(1)$ & $37(1)$ & $38(1)$ & $-6(1)$ & $-3(1)$ & $1(1)$ \\
\hline C12 & $68(1)$ & 49 (1) & $90(2)$ & $-25(1)$ & $-25(1)$ & $-6(1)$ \\
\hline 04 & $88(1)$ & $42(1)$ & $59(1)$ & $-16(1)$ & $0(1)$ & $-18(1)$ \\
\hline 06 & $112(1)$ & $57(1)$ & $37(1)$ & $-8(1)$ & $10(1)$ & $-26(1)$ \\
\hline 09 & $101(1)$ & $46(1)$ & $97(1)$ & $-24(1)$ & $-2(1)$ & $11(1)$ \\
\hline
\end{tabular}


Theoretical data for compound 1 :

Final Energy in Hartrees: HF $=-879.7282735$ (B3LYP / 6-31++G(D,P))

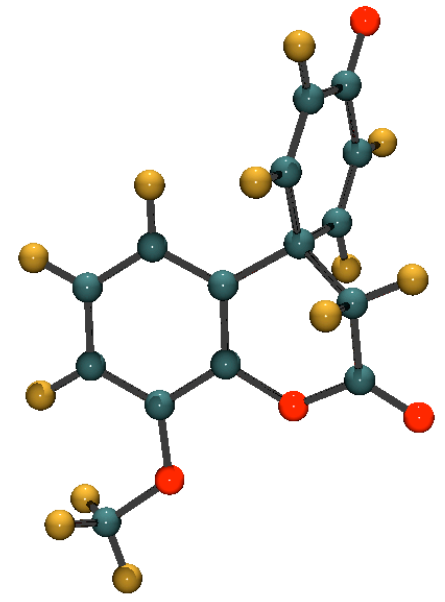

Standard orientation:

\begin{tabular}{|c|c|c|c|c|c|}
\hline \multirow{2}{*}{$\begin{array}{l}\text { Center } \\
\text { Number }\end{array}$} & \multirow{2}{*}{$\begin{array}{l}\text { Atomic } \\
\text { Number }\end{array}$} & \multirow{2}{*}{$\begin{array}{l}\text { Atomic } \\
\text { Type }\end{array}$} & \multicolumn{3}{|c|}{ Coordinates (Angstroms) } \\
\hline & & & $\mathrm{X}$ & $\mathrm{Y}$ & Z \\
\hline 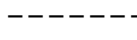 & & & & --- & --------- \\
\hline 1 & 6 & 0 & 1.430375 & -2.624924 & .213216 \\
\hline 2 & 6 & 0 & 2.621041 & -1.922539 & -.002833 \\
\hline 3 & 6 & 0 & 2.603370 & -.527822 & -.102583 \\
\hline 4 & 6 & 0 & 1.368559 & .143881 & .022208 \\
\hline 5 & 6 & 0 & .180569 & -.551790 & .249198 \\
\hline 6 & 6 & 0 & .219787 & -1.951149 & .338540 \\
\hline 7 & 8 & 0 & 3.690478 & .256180 & -.315312 \\
\hline 8 & 6 & 0 & 4.962127 & -.369795 & -.457047 \\
\hline 9 & 8 & 0 & 1.413634 & 1.520834 & -.149536 \\
\hline 10 & 6 & 0 & .385244 & 2.322836 & .266576 \\
\hline 11 & 6 & 0 & -.746564 & 1.629288 & .991570 \\
\hline 12 & 6 & 0 & -1.122870 & .252859 & .360784 \\
\hline 13 & 6 & 0 & -2.109260 & -.417821 & 1.284057 \\
\hline 14 & 6 & 0 & -3.357675 & -.758168 & .928875 \\
\hline 15 & 6 & 0 & -3.870456 & -.529416 & -.441128 \\
\hline 16 & 6 & 0 & -2.933292 & .107363 & -1.390807 \\
\hline 17 & 6 & 0 & -1.690638 & .463430 & -1.028670 \\
\hline 18 & 8 & 0 & -5.011154 & -.852488 & -.767131 \\
\hline 19 & 8 & 0 & .446034 & 3.507930 & .056338 \\
\hline 20 & 1 & 0 & 5.670075 & .442406 & -.622930 \\
\hline 21 & 1 & 0 & 4.977208 & -1.049835 & -1.317395 \\
\hline 22 & 1 & 0 & 5.239930 & -.917561 & .451778 \\
\hline 23 & 1 & 0 & -3.304543 & .271909 & -2.398013 \\
\hline 24 & 1 & 0 & -4.046438 & -1.221414 & 1.629455 \\
\hline 25 & 1 & 0 & -1.025817 & .928860 & -1.754349 \\
\hline 26 & 1 & 0 & -1.600472 & 2.307732 & 1.011579 \\
\hline 27 & 1 & 0 & -.417967 & 1.462243 & 2.026169 \\
\hline 28 & 1 & 0 & -1.756578 & -.606753 & 2.296725 \\
\hline 29 & 1 & 0 & 1.457016 & -3.707770 & .283683 \\
\hline 30 & 1 & 0 & -.700312 & -2.502051 & .501309 \\
\hline 31 & 1 & 0 & 3.554074 & -2.464804 & -.094619 \\
\hline
\end{tabular}

\begin{tabular}{lrr} 
& & \multicolumn{1}{c}{1} \\
& & \multicolumn{1}{c}{ A } \\
Frequencies -- & 37.9740 \\
Red. masses -- & 5.8775 \\
Frc consts -- & .0050 \\
IR Inten -- & 1.4632
\end{tabular}

2
A
43.9304
6.0358
.0069
1.7113

3

A

80.4251

4.2839

.0163

4.0625 
Theoretical data for compound 10 :

Final Energy in Hartrees: HF $=-879.1713081(B 3 L Y P / 6-31++G(D, P))$

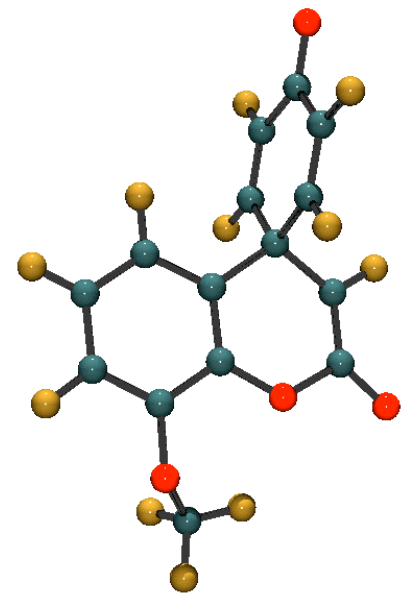

Standard orientation:

\begin{tabular}{|c|c|c|c|c|c|}
\hline \multirow{2}{*}{$\begin{array}{l}\text { Center } \\
\text { Number }\end{array}$} & \multirow{2}{*}{$\begin{array}{l}\text { Atomic } \\
\text { Number }\end{array}$} & \multirow{2}{*}{$\begin{array}{c}\text { Atomic } \\
\text { Type }\end{array}$} & \multicolumn{3}{|c|}{ Coordinates (Angstroms) } \\
\hline & & & $\mathrm{X}$ & $\mathrm{Y}$ & Z \\
\hline 1 & 6 & 0 & -1.099823 & -2.852971 & .067652 \\
\hline 2 & 6 & 0 & -2.382061 & -2.323520 & -.112456 \\
\hline 3 & 6 & 0 & -2.568667 & -.946705 & -.215622 \\
\hline 4 & 6 & 0 & -1.456732 & -.068011 & -.152804 \\
\hline 5 & 6 & 0 & -.171113 & -.599612 & .052513 \\
\hline 6 & 6 & 0 & -.009651 & -1.988789 & .155230 \\
\hline 7 & 8 & 0 & -1.706282 & 1.247676 & -.317863 \\
\hline 8 & 6 & 0 & -.685634 & 2.260225 & .026098 \\
\hline 9 & 6 & 0 & .584949 & 1.782350 & .281556 \\
\hline 10 & 6 & 0 & 1.040592 & .337881 & .110827 \\
\hline 11 & 6 & 0 & 1.818158 & .255868 & -1.185026 \\
\hline 12 & 6 & 0 & 3.149086 & .036962 & -1.268887 \\
\hline 13 & 6 & 0 & 3.956172 & -.240459 & -.072257 \\
\hline 14 & 6 & 0 & 3.235488 & -.250753 & 1.217840 \\
\hline 15 & 6 & 0 & 1.910831 & -.017958 & 1.292528 \\
\hline 16 & 8 & 0 & 5.175445 & -.458782 & -.133048 \\
\hline 17 & 8 & 0 & -1.154866 & 3.403836 & .040157 \\
\hline 18 & 8 & 0 & -3.848313 & -.491805 & -.457807 \\
\hline 19 & 6 & 0 & -4.385441 & .456529 & .474592 \\
\hline 20 & 1 & 0 & -5.418225 & .626898 & .159525 \\
\hline 21 & 1 & 0 & -3.824605 & 1.393258 & .457413 \\
\hline 22 & 1 & 0 & -4.381607 & .035402 & 1.489925 \\
\hline 23 & 1 & 0 & 3.837077 & -.447814 & 2.101758 \\
\hline 24 & 1 & 0 & 3.680910 & .063099 & -2.216596 \\
\hline 25 & 1 & 0 & 1.399192 & -.019641 & 2.252713 \\
\hline 26 & 1 & 0 & 1.352664 & 2.517073 & .492073 \\
\hline 27 & 1 & 0 & 1.233079 & .457160 & -2.079765 \\
\hline 28 & 1 & 0 & -.957141 & -3.927409 & .143763 \\
\hline 29 & 1 & 0 & .991046 & -2.388123 & .297472 \\
\hline 30 & 1 & 0 & -3.257177 & -2.963526 & -.180502 \\
\hline
\end{tabular}

\begin{tabular}{|c|c|c|c|c|}
\hline & & 1 & 2 & 3 \\
\hline & & A & A & A \\
\hline Frequencies & -- & 23.6524 & 50.0833 & 77.0913 \\
\hline Red. masses & -- & 5.1137 & 8.2316 & 3.8149 \\
\hline Frc consts & -- & .0017 & .0122 & .0134 \\
\hline IR Inten & -- & .1974 & 2.6776 & 3.0598 \\
\hline
\end{tabular}


Theoretical data for transition state TS1:

Final Energy in Hartrees: HF $=-879.1565668(B 3 L Y P / 6-31++G(D, P))$

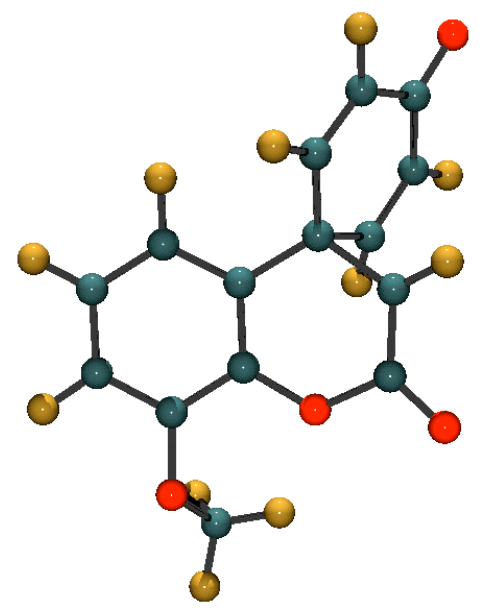

Standard orientation:

\begin{tabular}{|c|c|c|c|c|c|}
\hline \multirow{2}{*}{$\begin{array}{l}\text { Center } \\
\text { Number }\end{array}$} & \multirow{2}{*}{$\begin{array}{l}\text { Atomic } \\
\text { Number }\end{array}$} & \multirow{2}{*}{$\begin{array}{c}\text { Atomic } \\
\text { Type }\end{array}$} & \multicolumn{3}{|c|}{ Coordinates (Angstroms) } \\
\hline & & & $\mathrm{X}$ & $\mathrm{Y}$ & Z \\
\hline 1 & 6 & 0 & 1.762037 & 2.812218 & -.089311 \\
\hline 2 & 6 & 0 & 2.882876 & 1.989731 & .035655 \\
\hline 3 & 6 & 0 & 2.743113 & .600062 & .021253 \\
\hline 4 & 6 & 0 & 1.462768 & .027827 & -.127712 \\
\hline 5 & 6 & 0 & .328060 & .850609 & -.234145 \\
\hline 6 & 6 & 0 & .494473 & 2.241037 & -.217040 \\
\hline 7 & 8 & 0 & 1.393892 & -1.341116 & -.153544 \\
\hline 8 & 6 & 0 & .242067 & -2.020849 & -.673900 \\
\hline 9 & 6 & 0 & -.966746 & -1.285037 & -.689980 \\
\hline 10 & 6 & 0 & -1.027367 & .208614 & -.334931 \\
\hline 11 & 6 & 0 & -2.091101 & .992244 & -1.026739 \\
\hline 12 & 6 & 0 & -3.378996 & .932594 & -.641946 \\
\hline 13 & 6 & 0 & -3.872450 & .133695 & .524643 \\
\hline 14 & 6 & 0 & -2.875262 & -.564137 & 1.249143 \\
\hline 15 & 6 & 0 & -1.507795 & -.551482 & .858863 \\
\hline 16 & 8 & 0 & -5.107060 & .159937 & .789692 \\
\hline 17 & 8 & 0 & .439835 & -3.184879 & -1.001810 \\
\hline 18 & 8 & 0 & 3.892712 & -.159296 & .074873 \\
\hline 19 & 6 & 0 & 4.001168 & -1.094708 & 1.154339 \\
\hline 20 & 1 & 0 & 5.003775 & -1.522315 & 1.076342 \\
\hline 21 & 1 & 0 & 3.251179 & -1.884599 & 1.074182 \\
\hline 22 & 1 & 0 & 3.900847 & -.578728 & 2.119345 \\
\hline 23 & 1 & 0 & -3.175830 & -1.148391 & 2.113975 \\
\hline 24 & 1 & 0 & -4.145830 & 1.488939 & -1.177672 \\
\hline 25 & 1 & 0 & -.750657 & -.833117 & 1.584782 \\
\hline 26 & 1 & 0 & -1.804899 & -1.721033 & -1.214428 \\
\hline 27 & 1 & 0 & -1.786970 & 1.601024 & -1.877644 \\
\hline 28 & 1 & 0 & 1.874710 & 3.892732 & -.072820 \\
\hline 29 & 1 & 0 & -.385208 & 2.873298 & -.287786 \\
\hline 30 & 1 & 0 & 3.882775 & 2.400510 & .138889 \\
\hline
\end{tabular}

\begin{tabular}{|c|c|c|c|}
\hline & 1 & 2 & 3 \\
\hline & A & A & A \\
\hline Frequencies & $--\quad-306.1658$ & 47.2981 & 70.6809 \\
\hline Red. masses & 10.1525 & 5.5042 & 7.5343 \\
\hline Frc consts & .5607 & .0073 & .0222 \\
\hline IR Inten & 679.7455 & 1.4875 & 5.1084 \\
\hline
\end{tabular}


Theoretical data for compound 11 :

Final Energy in Hartrees: HF $=-879.1575149$ (B3LYP / 6-31++G(D,P))

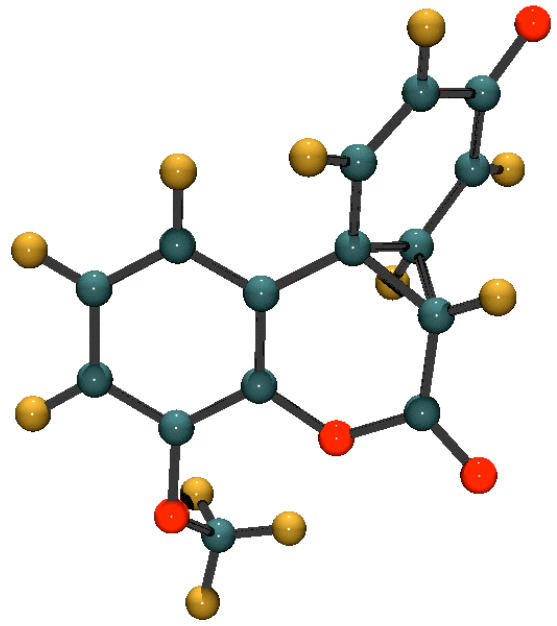

Standard orientation:

\begin{tabular}{|c|c|c|c|c|c|}
\hline \multirow{2}{*}{$\begin{array}{l}\text { Center } \\
\text { Number }\end{array}$} & \multirow{2}{*}{$\begin{array}{l}\text { Atomic } \\
\text { Number }\end{array}$} & \multirow{2}{*}{$\begin{array}{c}\text { Atomic } \\
\text { Type }\end{array}$} & \multicolumn{3}{|c|}{ Coordinates (Angstroms) } \\
\hline & & & $\mathrm{X}$ & $\mathrm{Y}$ & Z \\
\hline 1 & 6 & 0 & 1.830040 & 2.797463 & -.071724 \\
\hline 2 & 6 & 0 & 2.932000 & 1.949851 & .050594 \\
\hline 3 & 6 & 0 & 2.757611 & .563768 & .022794 \\
\hline 4 & 6 & 0 & 1.465679 & .028664 & -.139005 \\
\hline 5 & 6 & 0 & .346388 & .872614 & -.239086 \\
\hline 6 & 6 & 0 & .549154 & 2.259542 & -.207675 \\
\hline 7 & 8 & 0 & 1.371328 & -1.345710 & -.197197 \\
\hline 8 & 6 & 0 & .208904 & -1.988554 & -.679070 \\
\hline 9 & 6 & 0 & -1.022991 & -1.249849 & -.603895 \\
\hline 10 & 6 & 0 & -1.017239 & .273691 & -.341464 \\
\hline 11 & 6 & 0 & -2.088583 & 1.067370 & -.991238 \\
\hline 12 & 6 & 0 & -3.378785 & .956625 & -.615073 \\
\hline 13 & 6 & 0 & -3.869451 & .118146 & .524238 \\
\hline 14 & 6 & 0 & -2.872787 & -.587109 & 1.222433 \\
\hline 15 & 6 & 0 & -1.482653 & -.602843 & .805574 \\
\hline 16 & 8 & 0 & -5.110250 & .150989 & .795114 \\
\hline 17 & 8 & 0 & .360958 & -3.135659 & -1.066143 \\
\hline 18 & 8 & 0 & 3.884996 & -.228376 & .074596 \\
\hline 19 & 6 & 0 & 3.972050 & -1.151124 & 1.166408 \\
\hline 20 & 1 & 0 & 4.958058 & -1.615034 & 1.085956 \\
\hline 21 & 1 & 0 & 3.195582 & -1.917220 & 1.104707 \\
\hline 22 & 1 & 0 & 3.897988 & -.618852 & 2.124710 \\
\hline 23 & 1 & 0 & -3.152015 & -1.154191 & 2.106032 \\
\hline 24 & 1 & 0 & -4.151057 & 1.527222 & -1.128143 \\
\hline 25 & 1 & 0 & -.737753 & -.805479 & 1.573245 \\
\hline 26 & 1 & 0 & -1.840061 & -1.655617 & -1.186229 \\
\hline 27 & 1 & 0 & -1.795939 & 1.735808 & -1.800652 \\
\hline 28 & 1 & 0 & 1.967395 & 3.874928 & -.044685 \\
\hline 29 & 1 & 0 & -.315072 & 2.912970 & -.270753 \\
\hline 30 & 1 & 0 & 3.940597 & 2.335985 & .161980 \\
\hline
\end{tabular}

\begin{tabular}{|c|c|c|c|c|}
\hline & & 1 & 2 & 3 \\
\hline & & A & A & A \\
\hline Frequencies & -- & 48.2311 & 70.1956 & 82.0859 \\
\hline Red. masses & -- & 5.6904 & 7.1232 & 2.1130 \\
\hline Frc consts & -- & .0078 & .0207 & .0084 \\
\hline IR Inten & -- & 1.5936 & 4.5749 & 2.4441 \\
\hline
\end{tabular}


Theoretical data for transition state TS2 :

Final Energy in Hartrees: HF = - 879.1498774 (B3LYP/6-31++G(D,P))

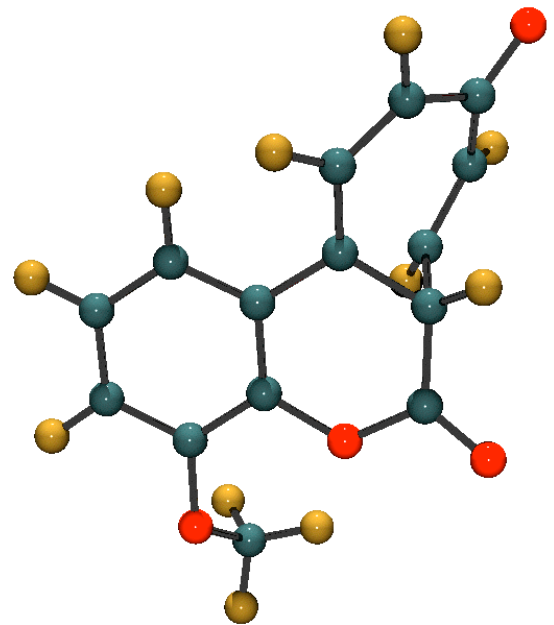

Standard orientation:

\begin{tabular}{|c|c|c|c|c|c|}
\hline \multirow{2}{*}{$\begin{array}{l}\text { Center } \\
\text { Number }\end{array}$} & \multirow{2}{*}{$\begin{array}{l}\text { Atomic } \\
\text { Number }\end{array}$} & \multirow{2}{*}{$\begin{array}{c}\text { Atomic } \\
\text { Type }\end{array}$} & \multicolumn{3}{|c|}{ Coordinates (Angstroms) } \\
\hline & & & $\mathrm{X}$ & $\mathrm{Y}$ & Z \\
\hline 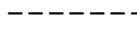 & & & & & --------- \\
\hline 1 & 6 & 0 & 1.991332 & 2.759143 & .028772 \\
\hline 2 & 6 & 0 & 3.054286 & 1.856076 & .107168 \\
\hline 3 & 6 & 0 & 2.801733 & .482672 & .004455 \\
\hline 4 & 6 & 0 & 1.490466 & .032580 & -.194103 \\
\hline 5 & 6 & 0 & .400029 & .925571 & -.257191 \\
\hline 6 & 6 & 0 & .684811 & 2.301430 & -.133744 \\
\hline 7 & 8 & 0 & 1.332975 & -1.343693 & -.345028 \\
\hline 8 & 6 & 0 & .117357 & -1.919713 & -.663535 \\
\hline 9 & 6 & 0 & -1.102501 & -1.100030 & -.511240 \\
\hline 10 & 6 & 0 & -.956278 & .404500 & -.408237 \\
\hline 11 & 6 & 0 & -2.049201 & 1.221933 & -.891606 \\
\hline 12 & 6 & 0 & -3.364550 & .969723 & -.625544 \\
\hline 13 & 6 & 0 & -3.886536 & .085363 & .441455 \\
\hline 14 & 6 & 0 & -2.893935 & -.532625 & 1.256949 \\
\hline 15 & 6 & 0 & -1.537099 & -.701408 & .874813 \\
\hline 16 & 8 & 0 & -5.131875 & .082607 & .687464 \\
\hline 17 & 8 & 0 & .137008 & -3.076820 & -1.028650 \\
\hline 18 & 8 & 0 & 3.880871 & -.379087 & .021835 \\
\hline 19 & 6 & 0 & 3.923296 & -1.316361 & 1.101854 \\
\hline 20 & 1 & 0 & 4.873879 & -1.846231 & 1.002370 \\
\hline 21 & 1 & 0 & 3.097461 & -2.030844 & 1.045665 \\
\hline 22 & 1 & 0 & 3.898385 & -.792178 & 2.067086 \\
\hline 23 & 1 & 0 & -3.164653 & -.766447 & 2.283432 \\
\hline 24 & 1 & 0 & -4.125681 & 1.592774 & -1.092193 \\
\hline 25 & 1 & 0 & -.798758 & -.880922 & 1.655018 \\
\hline 26 & 1 & 0 & -1.905465 & -1.460601 & -1.150695 \\
\hline 27 & 1 & 0 & -1.785898 & 2.093272 & -1.492571 \\
\hline 28 & 1 & 0 & 2.181389 & 3.825697 & .117190 \\
\hline 29 & 1 & 0 & -.141325 & 3.004531 & -.147085 \\
\hline 30 & 1 & 0 & 4.079582 & 2.183773 & .245220 \\
\hline
\end{tabular}

\begin{tabular}{|c|c|c|c|c|}
\hline & & 1 & 2 & 3 \\
\hline & & A & A & A \\
\hline Frequencies & -- & -490.6418 & 45.5429 & 69.6040 \\
\hline Red. masses & -- & 5.3992 & 5.8733 & 3.8492 \\
\hline Frc consts & -- & .7658 & .0072 & .0110 \\
\hline IR Inten & -- & 192.1547 & 1.7354 & 5.4452 \\
\hline
\end{tabular}


Theoretical data for compound $12 \mathrm{c}$ :

Final Energy in Hartrees: HF $=-879.1830086(B 3 L Y P / 6-31++G(D, P))$

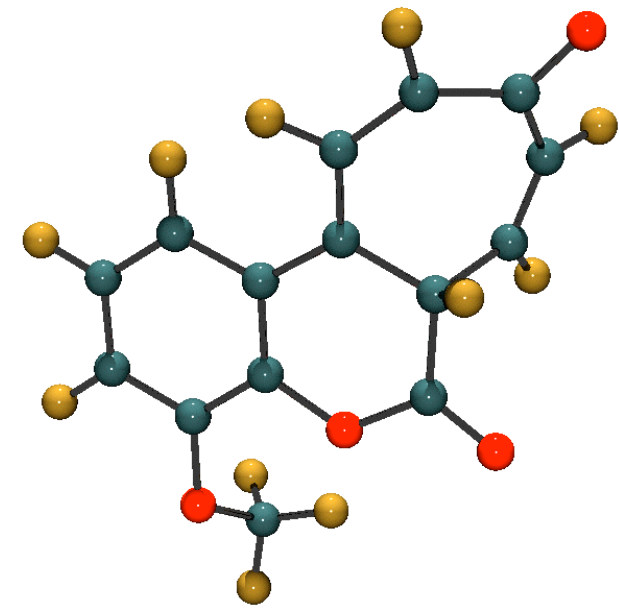

Standard orientation:

\begin{tabular}{|c|c|c|c|c|c|}
\hline \multirow{2}{*}{$\begin{array}{l}\text { Center } \\
\text { Number }\end{array}$} & \multirow{2}{*}{$\begin{array}{l}\text { Atomic } \\
\text { Number }\end{array}$} & \multirow{2}{*}{$\begin{array}{c}\text { Atomic } \\
\text { Type }\end{array}$} & \multicolumn{3}{|c|}{ Coordinates (Angstroms) } \\
\hline & & & $\mathrm{X}$ & $\mathrm{Y}$ & Z \\
\hline 1 & 6 & 0 & 2.335577 & 2.677858 & .251889 \\
\hline 2 & 6 & 0 & 3.320110 & 1.687280 & .145429 \\
\hline 3 & 6 & 0 & 2.924765 & .363419 & -.074728 \\
\hline 4 & 6 & 0 & 1.566548 & .056256 & -.205162 \\
\hline 5 & 6 & 0 & .546117 & 1.031948 & -.100438 \\
\hline 6 & 6 & 0 & .985961 & 2.359914 & .153063 \\
\hline 7 & 8 & 0 & 1.270963 & -1.277981 & -.477378 \\
\hline 8 & 6 & 0 & .000414 & -1.775410 & -.507299 \\
\hline 9 & 6 & 0 & -1.175356 & -.831733 & -.346487 \\
\hline 10 & 6 & 0 & -.827778 & .640135 & -.267227 \\
\hline 11 & 6 & 0 & -1.875458 & 1.538939 & -.453509 \\
\hline 12 & 6 & 0 & -3.256571 & 1.288041 & -.465608 \\
\hline 13 & 6 & 0 & -3.993232 & .222438 & .160908 \\
\hline 14 & 6 & 0 & -3.278597 & -.771295 & 1.011439 \\
\hline 15 & 6 & 0 & -2.035032 & -1.250326 & .833021 \\
\hline 16 & 8 & 0 & -5.252903 & .172737 & .137627 \\
\hline 17 & 8 & 0 & -.122864 & -2.970504 & -.679653 \\
\hline 18 & 8 & 0 & 3.909685 & -.595509 & -.232150 \\
\hline 19 & 6 & 0 & 3.951887 & -1.618693 & .765245 \\
\hline 20 & 1 & 0 & 2.629018 & 3.708089 & .439438 \\
\hline 21 & 1 & 0 & 4.378441 & 1.909370 & .232365 \\
\hline 22 & 1 & 0 & .243072 & 3.138436 & .288234 \\
\hline 23 & 1 & 0 & -1.791455 & -.990033 & -1.251905 \\
\hline 24 & 1 & 0 & -1.593787 & 2.572195 & -.664226 \\
\hline 25 & 1 & 0 & -3.899675 & 2.072967 & -.860299 \\
\hline 26 & 1 & 0 & -3.874991 & -1.140038 & 1.844359 \\
\hline 27 & 1 & 0 & -1.618152 & -1.963356 & 1.543020 \\
\hline 28 & 1 & 0 & 4.830967 & -2.226972 & .536677 \\
\hline 29 & 1 & 0 & 3.055942 & -2.245485 & .736111 \\
\hline 30 & 1 & 0 & 4.065440 & -1.177740 & 1.765442 \\
\hline
\end{tabular}

\begin{tabular}{|c|c|c|c|c|}
\hline & & 1 & 2 & 3 \\
\hline & & A & A & A \\
\hline Frequencies & -- & 35.6304 & 62.8939 & 78.0753 \\
\hline Red. masses & -- & 6.7930 & 3.5666 & 6.1076 \\
\hline Frc consts & -- & .0051 & .0083 & .0219 \\
\hline IR Inten & -- & .2839 & .3609 & 3.1001 \\
\hline
\end{tabular}


Theoretical data for compound 2a :

Final Energy in Hartrees: HF $=-879.7472803$ (B3LYP / 6-31++G(D,P))

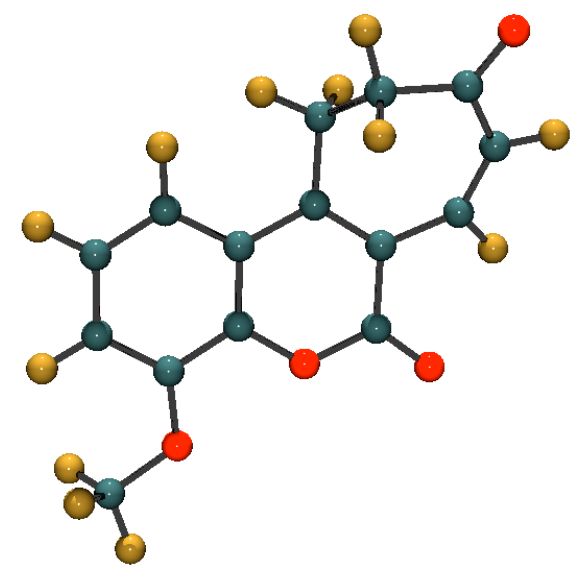

Standard orientation:

\begin{tabular}{|c|c|c|c|c|c|}
\hline \multirow{2}{*}{$\begin{array}{l}\text { Center } \\
\text { Number }\end{array}$} & \multirow{2}{*}{$\begin{array}{l}\text { Atomic } \\
\text { Number }\end{array}$} & \multirow{2}{*}{$\begin{array}{c}\text { Atomic } \\
\text { Type }\end{array}$} & \multicolumn{3}{|c|}{ Coordinates (Angstroms) } \\
\hline & & & $\mathrm{X}$ & $\mathrm{Y}$ & Z \\
\hline & & & & -------1 & ---------- \\
\hline 1 & 6 & 0 & 2.349150 & -2.507020 & -.140323 \\
\hline 2 & 6 & 0 & 3.310763 & -1.496038 & .021122 \\
\hline 3 & 6 & 0 & 2.917250 & -.160087 & .074401 \\
\hline 4 & 6 & 0 & 1.540855 & .145310 & -.038901 \\
\hline 5 & 6 & 0 & .566571 & -.854500 & -.204577 \\
\hline 6 & 6 & 0 & 1.004075 & -2.202037 & -.250928 \\
\hline 7 & 8 & 0 & 3.747390 & .900227 & .228288 \\
\hline 8 & 6 & 0 & 5.145697 & .654887 & .347793 \\
\hline 9 & 8 & 0 & 1.219489 & 1.464892 & .017180 \\
\hline 10 & 6 & 0 & -.079672 & 1.920359 & -.070011 \\
\hline 11 & 6 & 0 & -1.139629 & .904328 & -.225502 \\
\hline 12 & 6 & 0 & -.823046 & -.433999 & -.310222 \\
\hline 13 & 8 & 0 & -.255921 & 3.118293 & -.015285 \\
\hline 14 & 6 & 0 & -2.474584 & 1.483322 & -.363343 \\
\hline 15 & 6 & 0 & -3.709570 & .954388 & -.180142 \\
\hline 16 & 6 & 0 & -4.085459 & -.382297 & .318918 \\
\hline 17 & 6 & 0 & -3.010718 & -1.432930 & .550618 \\
\hline 18 & 6 & 0 & -1.918926 & -1.445294 & -.537072 \\
\hline 19 & 8 & 0 & -5.262011 & -.636784 & .557367 \\
\hline 20 & 1 & 0 & 5.605419 & 1.636875 & .459401 \\
\hline 21 & 1 & 0 & 5.541581 & .165065 & -.550198 \\
\hline 22 & 1 & 0 & 5.368002 & .043875 & 1.231139 \\
\hline 23 & 1 & 0 & -3.519087 & -2.399541 & .599453 \\
\hline 24 & 1 & 0 & -2.563546 & -1.255830 & 1.538860 \\
\hline 25 & 1 & 0 & -4.566968 & 1.602253 & -.342670 \\
\hline 26 & 1 & 0 & 2.671876 & -3.542588 & -.176273 \\
\hline 27 & 1 & 0 & .290442 & -3.006791 & -.368260 \\
\hline 28 & 1 & 0 & 4.357227 & -1.762248 & .105044 \\
\hline 29 & 1 & 0 & -2.441191 & 2.538150 & -.623357 \\
\hline 30 & 1 & 0 & -1.508287 & -2.450502 & -.613372 \\
\hline 31 & 1 & 0 & -2.383598 & -1.232090 & -1.511147 \\
\hline
\end{tabular}

\begin{tabular}{|c|c|c|c|c|}
\hline & & 1 & 2 & 3 \\
\hline & & A & A & A \\
\hline Frequencies & -- & 31.7646 & 53.9631 & 71.6721 \\
\hline Red. masses & -- & 6.0848 & 3.7000 & 6.2170 \\
\hline Frc consts & -- & .0036 & .0063 & .0188 \\
\hline IR Inten & -- & .5194 & 1.4801 & 10.6039 \\
\hline
\end{tabular}


Theoretical data for transition state TS4n :

Final Energy in Hartrees: $\mathrm{HF}=-879.662717$ (B3LYP/6-31++G(D,P))

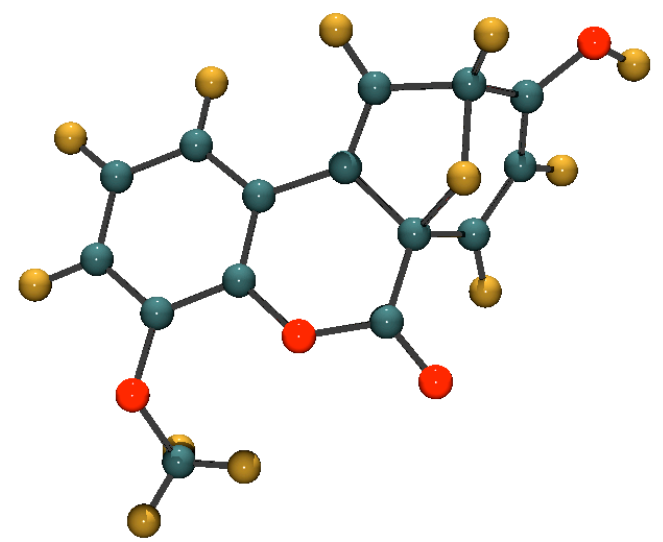

Standard orientation:

\begin{tabular}{|c|c|c|c|c|c|}
\hline \multirow{3}{*}{$\begin{array}{l}\text { Center } \\
\text { Number }\end{array}$} & \multirow{2}{*}{$\begin{array}{l}\text { Atomic } \\
\text { Number }\end{array}$} & \multirow{2}{*}{$\begin{array}{c}\text { Atomic } \\
\text { Type }\end{array}$} & \multicolumn{3}{|c|}{ Coordinates (Angstroms) } \\
\hline & & & $\mathrm{X}$ & $\mathrm{Y}$ & Z \\
\hline & 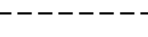 & & & & --------- \\
\hline 1 & 6 & 0 & 2.424677 & 2.655365 & .403496 \\
\hline 2 & 6 & 0 & 3.365309 & 1.636139 & .230276 \\
\hline 3 & 6 & 0 & 2.958324 & .333511 & -.071641 \\
\hline 4 & 6 & 0 & 1.583637 & .072055 & -.209371 \\
\hline 5 & 6 & 0 & .627554 & 1.086422 & -.036927 \\
\hline 6 & 6 & 0 & 1.064765 & 2.383114 & .280003 \\
\hline 7 & 8 & 0 & 3.925313 & -.606824 & -.304929 \\
\hline 8 & 6 & 0 & 3.920715 & -1.789692 & .511612 \\
\hline 9 & 8 & 0 & 1.226437 & -1.217005 & -.577833 \\
\hline 10 & 6 & 0 & -.045409 & -1.718288 & -.387352 \\
\hline 11 & 6 & 0 & -1.134013 & -.736911 & -.117287 \\
\hline 12 & 6 & 0 & -.765191 & .714782 & -.272374 \\
\hline 13 & 8 & 0 & -.198716 & -2.916255 & -.461189 \\
\hline 14 & 6 & 0 & -1.978092 & -1.095991 & .997645 \\
\hline 15 & 6 & 0 & -3.218478 & -.519000 & 1.190716 \\
\hline 16 & 6 & 0 & -3.804525 & .229514 & .145843 \\
\hline 17 & 6 & 0 & -3.044839 & .683018 & -.964734 \\
\hline 18 & 6 & 0 & -1.796930 & 1.458880 & -.711085 \\
\hline 19 & 8 & 0 & -5.150946 & .383565 & .076332 \\
\hline 20 & 1 & 0 & 2.757488 & 3.659527 & .647334 \\
\hline 21 & 1 & 0 & 4.429509 & 1.826154 & .323039 \\
\hline 22 & 1 & 0 & .329525 & 3.166585 & .433258 \\
\hline 23 & 1 & 0 & 4.839297 & -2.321641 & .259030 \\
\hline 24 & 1 & 0 & 3.055701 & -2.420834 & .297554 \\
\hline 25 & 1 & 0 & 3.937192 & -1.518258 & 1.574473 \\
\hline 26 & 1 & 0 & -1.678539 & -1.919488 & 1.641991 \\
\hline 27 & 1 & 0 & -3.830841 & -.829895 & 2.034151 \\
\hline 28 & 1 & 0 & -3.653422 & 1.001031 & -1.812975 \\
\hline 29 & 1 & 0 & -2.200999 & -.527817 & -1.028892 \\
\hline 30 & 1 & 0 & -1.755042 & 2.523330 & -.919649 \\
\hline 31 & 1 & 0 & -5.591618 & -.144047 & .758723 \\
\hline
\end{tabular}

1

A

Frequencies -- -1503.8333

Red. masses -- 1.1994

Frc consts -- 1.5982

IR Inten -- 247.0457

\section{2}

A

43.5221

6.1536

.0069

1.7397
3

A

58.9796

2.0421

.0042

2.5080 
Theoretical data for transition state TS4a :

Final Energy in Hartrees: HF $=-879.1443616$ (B3LYP / 6-31++G(D,P))

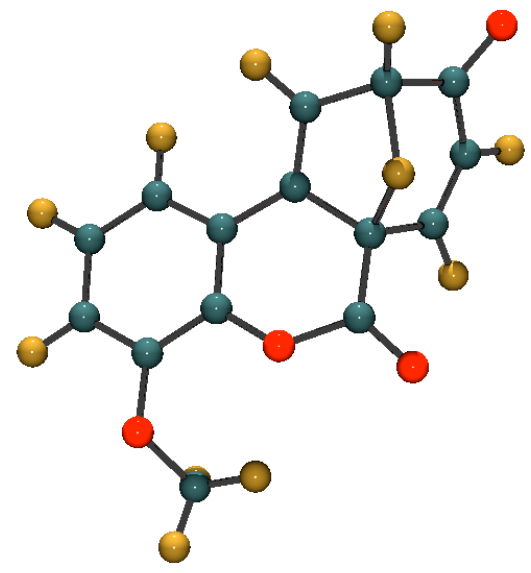

Standard orientation:

\begin{tabular}{|c|c|c|c|c|c|}
\hline \multirow{2}{*}{$\begin{array}{l}\text { Center } \\
\text { Number }\end{array}$} & \multirow{2}{*}{$\begin{array}{l}\text { Atomic } \\
\text { Number }\end{array}$} & \multirow{2}{*}{$\begin{array}{c}\text { Atomic } \\
\text { Type }\end{array}$} & \multicolumn{3}{|c|}{ Coordinates (Angstroms) } \\
\hline & & & $\mathrm{X}$ & $\mathrm{Y}$ & Z \\
\hline & & & & $0<19020$ & ------- \\
\hline 1 & 6 & 0 & 2.412460 & 2.644924 & .430127 \\
\hline 2 & 6 & 0 & 3.353816 & 1.631956 & .216492 \\
\hline 3 & 6 & 0 & 2.928919 & .336883 & -.097623 \\
\hline 4 & 6 & 0 & 1.554657 & .070975 & -.209893 \\
\hline 5 & 6 & 0 & .593066 & 1.077694 & .015001 \\
\hline 6 & 6 & 0 & 1.049229 & 2.369535 & .344228 \\
\hline 7 & 8 & 0 & 1.183408 & -1.199535 & -.595416 \\
\hline 8 & 6 & 0 & -.101695 & -1.709630 & -.334994 \\
\hline 9 & 6 & 0 & -1.180581 & -.741945 & -.100597 \\
\hline 10 & 6 & 0 & -.794613 & .703235 & -.184137 \\
\hline 11 & 6 & 0 & -1.821465 & 1.471174 & -.645435 \\
\hline 12 & 6 & 0 & -3.061427 & .760480 & -.968287 \\
\hline 13 & 6 & 0 & -3.948442 & .241275 & .087218 \\
\hline 14 & 6 & 0 & -3.329051 & -.586567 & 1.137662 \\
\hline 15 & 6 & 0 & -2.095239 & -1.130402 & .998668 \\
\hline 16 & 8 & 0 & -5.191362 & .388298 & .058649 \\
\hline 17 & 8 & 0 & -.199449 & -2.923404 & -.361573 \\
\hline 18 & 8 & 0 & 3.896761 & -.610292 & -.363527 \\
\hline 19 & 6 & 0 & 3.872910 & -1.797787 & .437693 \\
\hline 20 & 1 & 0 & 4.767889 & -2.360594 & .160465 \\
\hline 21 & 1 & 0 & 2.980158 & -2.396043 & .241864 \\
\hline 22 & 1 & 0 & 3.922400 & -1.540547 & 1.504732 \\
\hline 23 & 1 & 0 & -3.983720 & -.895326 & 1.949330 \\
\hline 24 & 1 & 0 & -3.650773 & 1.196020 & -1.780067 \\
\hline 25 & 1 & 0 & -1.763393 & -1.907039 & 1.687927 \\
\hline 26 & 1 & 0 & -2.174153 & -.523676 & -1.033214 \\
\hline 27 & 1 & 0 & -1.694158 & 2.522276 & -.900780 \\
\hline 28 & 1 & 0 & 2.748341 & 3.647488 & .682622 \\
\hline 29 & 1 & 0 & .316535 & 3.147455 & .536826 \\
\hline 30 & 1 & 0 & 4.420739 & 1.818642 & .288487 \\
\hline
\end{tabular}

1
A

Frequencies -- -1437.1718

Red. masses -- 1.1704

Frc consts -- 1.4243

IR Inten -- 543.4094
2

$\begin{array}{ll}45.6003 & 70.2999\end{array}$

$6.5841 \quad 2.5285$

$.0081 \quad .0074$

$.0748 \quad 2.2404$ 
Theoretical data for transition state TS5a :

Final Energy in Hartrees: HF $=-879.1087276(B 3 L Y P / 6-31++G(D, P))$

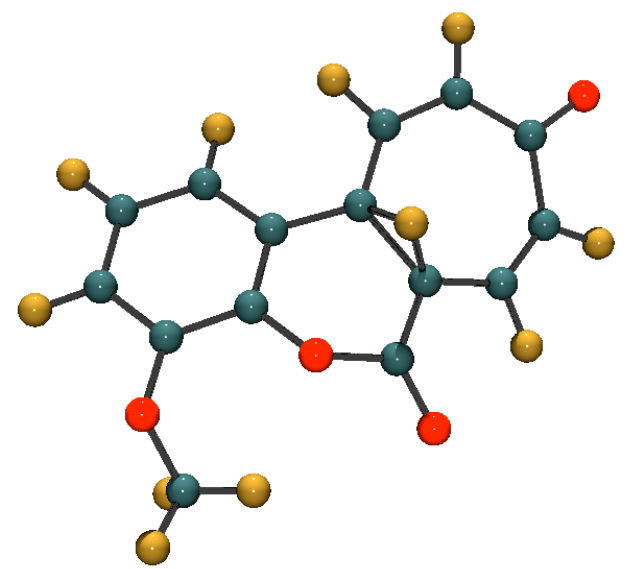

Standard orientation:

\begin{tabular}{|c|c|c|c|c|c|}
\hline \multirow{2}{*}{$\begin{array}{l}\text { Center } \\
\text { Number }\end{array}$} & \multirow{2}{*}{$\begin{array}{l}\text { Atomic } \\
\text { Number }\end{array}$} & \multirow{2}{*}{$\begin{array}{c}\text { Atomic } \\
\text { Type }\end{array}$} & \multicolumn{3}{|c|}{ Coordinates (Angstroms) } \\
\hline & & & $\mathrm{X}$ & $\mathrm{Y}$ & $\mathrm{Z}$ \\
\hline--- & ---- & ------ & ---------- & ----------1 & --------- \\
\hline 1 & 6 & 0 & 2.271007 & 2.709654 & .412532 \\
\hline 2 & 6 & 0 & 3.280412 & 1.758013 & .255286 \\
\hline 3 & 6 & 0 & 2.938058 & .439209 & -.073108 \\
\hline 4 & 6 & 0 & 1.593226 & .103722 & -.276729 \\
\hline 5 & 6 & 0 & .557432 & 1.048560 & -.114176 \\
\hline 6 & 6 & 0 & .928901 & 2.358775 & .257300 \\
\hline 7 & 8 & 0 & 1.304502 & -1.173721 & -.725645 \\
\hline 8 & 6 & 0 & .121902 & -1.800965 & -.236434 \\
\hline 9 & 6 & 0 & -1.031494 & -.957562 & -.148758 \\
\hline 10 & 6 & 0 & -.818397 & .598188 & -.318788 \\
\hline 11 & 6 & 0 & -1.903230 & 1.513478 & -.407375 \\
\hline 12 & 6 & 0 & -3.273487 & 1.380259 & -.328964 \\
\hline 13 & 6 & 0 & -4.074749 & .252673 & .124351 \\
\hline 14 & 6 & 0 & -3.505940 & -1.062873 & .520750 \\
\hline 15 & 6 & 0 & -2.249085 & -1.546704 & .376251 \\
\hline 16 & 8 & 0 & -5.312659 & .383026 & .221195 \\
\hline 17 & 8 & 0 & .208365 & -3.003177 & -.010553 \\
\hline 18 & 8 & 0 & 3.964686 & -.461151 & -.272376 \\
\hline 19 & 6 & 0 & 3.934253 & -1.650263 & .528321 \\
\hline 20 & 1 & 0 & 4.855368 & -2.189669 & .292622 \\
\hline 21 & 1 & 0 & 3.066320 & -2.270654 & .293977 \\
\hline 22 & 1 & 0 & 3.929500 & -1.390359 & 1.595948 \\
\hline 23 & 1 & 0 & -4.261567 & -1.726661 & .932504 \\
\hline 24 & 1 & 0 & -3.873832 & 2.257354 & -.555164 \\
\hline 25 & 1 & 0 & -2.089282 & -2.579159 & .685042 \\
\hline 26 & 1 & 0 & -1.006246 & -.291395 & -1.249489 \\
\hline 27 & 1 & 0 & -1.571081 & 2.529177 & -.623978 \\
\hline 28 & 1 & 0 & 2.529493 & 3.729904 & .685401 \\
\hline 29 & 1 & 0 & .159734 & 3.102305 & .435730 \\
\hline 30 & 1 & 0 & 4.329586 & 2.004359 & .383673 \\
\hline
\end{tabular}

\section{1 \\ A}

Frequencies -- -1653.2005

Red. masses -- 1.2708

Frc consts -- $\quad 2.0463$

IR Inten -- 1805.7267

2
A
47.5290
6.4905
.0086
1.0927

3

A

56.5620

4.2436

.0080

.2551 
Theoretical data for compound $\mathbf{2 b}$ :

Final Energy in Hartrees: HF $=-879.7303913$ (B3LYP / 6-31++G(D,P))

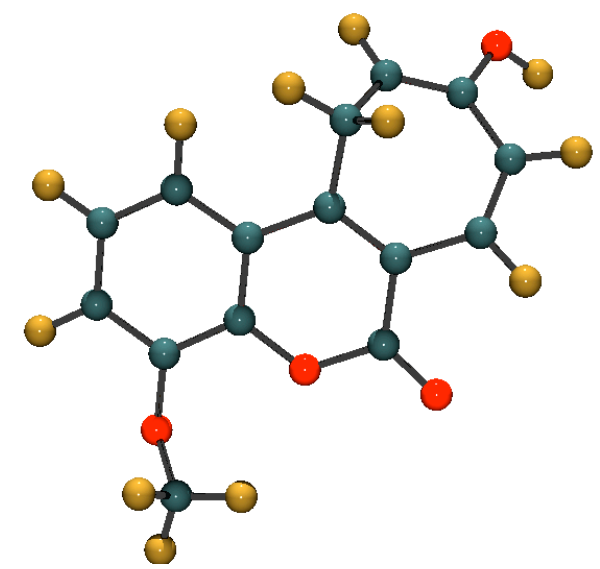

Standard orientation:

\begin{tabular}{|c|c|c|c|c|c|}
\hline \multirow{2}{*}{$\begin{array}{l}\text { Center } \\
\text { Number }\end{array}$} & \multirow{2}{*}{$\begin{array}{l}\text { Atomic } \\
\text { Number }\end{array}$} & \multirow{2}{*}{$\begin{array}{c}\text { Atomic } \\
\text { Type }\end{array}$} & \multicolumn{3}{|c|}{ Coordinates (Angstroms) } \\
\hline & & & $\mathrm{X}$ & $\mathrm{Y}$ & Z \\
\hline & 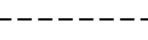 & & & --- & --------- \\
\hline 1 & 6 & 0 & -2.193964 & -2.717996 & -.005408 \\
\hline 2 & 6 & 0 & -3.183343 & -1.780092 & .316443 \\
\hline 3 & 6 & 0 & -2.885688 & -.419421 & .358380 \\
\hline 4 & 6 & 0 & -1.566146 & -.008887 & .083528 \\
\hline 5 & 6 & 0 & -.559332 & -.933911 & -.259383 \\
\hline 6 & 6 & 0 & -.902507 & -2.303378 & -.299425 \\
\hline 7 & 8 & 0 & -3.861281 & .453962 & .751821 \\
\hline 8 & 6 & 0 & -4.260214 & 1.473597 & -.181669 \\
\hline 9 & 8 & 0 & -1.309616 & 1.329381 & .163424 \\
\hline 10 & 6 & 0 & -.056619 & 1.873325 & -.060454 \\
\hline 11 & 6 & 0 & 1.030405 & .932030 & -.368538 \\
\hline 12 & 6 & 0 & .769101 & -.413274 & -.534670 \\
\hline 13 & 8 & 0 & .043640 & 3.080134 & .006075 \\
\hline 14 & 6 & 0 & 2.345896 & 1.538691 & -.480753 \\
\hline 15 & 6 & 0 & 3.539090 & .966586 & -.157270 \\
\hline 16 & 6 & 0 & 3.728781 & -.381598 & .348203 \\
\hline 17 & 6 & 0 & 2.944743 & -1.431612 & .015834 \\
\hline 18 & 6 & 0 & 1.888528 & -1.292406 & -1.048564 \\
\hline 19 & 8 & 0 & 4.794868 & -.595389 & 1.197666 \\
\hline 20 & 1 & 0 & -2.441307 & -3.774498 & -.027519 \\
\hline 21 & 1 & 0 & -4.197940 & -2.086821 & .548413 \\
\hline 22 & 1 & 0 & -.147791 & -3.042299 & -.539317 \\
\hline 23 & 1 & 0 & -5.107797 & 1.978518 & .283644 \\
\hline 24 & 1 & 0 & -4.579714 & 1.018045 & -1.126881 \\
\hline 25 & 1 & 0 & -3.455128 & 2.189550 & -.360269 \\
\hline 26 & 1 & 0 & 2.343896 & 2.596619 & -.725695 \\
\hline 27 & 1 & 0 & 4.424396 & 1.601378 & -.186119 \\
\hline 28 & 1 & 0 & 3.099226 & -2.386695 & .508575 \\
\hline 29 & 1 & 0 & 2.310219 & -.812661 & -1.943087 \\
\hline 30 & 1 & 0 & 1.521640 & -2.271618 & -1.354825 \\
\hline 31 & 1 & 0 & 5.057025 & .235852 & 1.613668 \\
\hline
\end{tabular}

\begin{tabular}{|c|c|c|c|c|}
\hline & & 1 & 2 & 3 \\
\hline & & A & A & A \\
\hline Frequencies & -- & 40.4893 & 59.3426 & 72.3116 \\
\hline Red. masses & -- & 6.2660 & 3.8561 & 2.0836 \\
\hline Frc consts & -- & .0061 & .0080 & .0064 \\
\hline IR Inten & -- & .6649 & .9673 & 3.7119 \\
\hline
\end{tabular}


Theoretical data for compound $2 \mathrm{c}$ :

Final Energy in Hartrees: HF $=-879.1790705$ (B3LYP / 6-31++G(D,P))

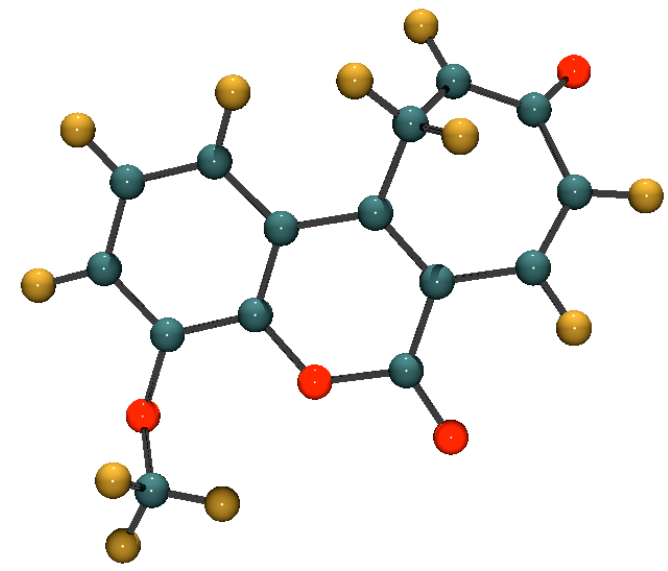

Standard orientation:

\begin{tabular}{|c|c|c|c|c|c|}
\hline \multirow{2}{*}{$\begin{array}{l}\text { Center } \\
\text { Number }\end{array}$} & \multirow{2}{*}{$\begin{array}{l}\text { Atomic } \\
\text { Number }\end{array}$} & \multirow{2}{*}{$\begin{array}{c}\text { Atomic } \\
\text { Type }\end{array}$} & \multicolumn{3}{|c|}{ Coordinates (Angstroms) } \\
\hline & & & $\mathrm{X}$ & $\mathrm{Y}$ & Z \\
\hline 1 & 6 & 0 & 2091416 & -2728786 & $=0$ \\
\hline $\begin{array}{l}1 \\
2\end{array}$ & 6 & 0 & 3.101241 & -1.811241 & -.318604 \\
\hline 3 & 6 & 0 & 2.817364 & -.446930 & -.358420 \\
\hline 4 & 6 & 0 & 1.508025 & -.006515 & -.085982 \\
\hline 5 & 6 & 0 & .483096 & -.912349 & .260714 \\
\hline 6 & 6 & 0 & .807200 & -2.287085 & .294118 \\
\hline 7 & 8 & 0 & 3.820644 & .416465 & -.742965 \\
\hline 8 & 6 & 0 & 4.204153 & 1.421210 & .203778 \\
\hline 9 & 8 & 0 & 1.279156 & 1.335755 & -.157830 \\
\hline 10 & 6 & 0 & .011239 & 1.902344 & .055428 \\
\hline 11 & 6 & 0 & -1.074543 & .991141 & .370540 \\
\hline 12 & 6 & 0 & -.828761 & -.368908 & .569537 \\
\hline 13 & 8 & 0 & -.034073 & 3.119157 & -.021290 \\
\hline 14 & 6 & 0 & -2.405141 & 1.566952 & .409224 \\
\hline 15 & 6 & 0 & -3.566346 & .937933 & .055781 \\
\hline 16 & 6 & 0 & -3.758193 & -.441988 & -.466559 \\
\hline 17 & 6 & 0 & -2.894069 & -1.443923 & .017289 \\
\hline 18 & 6 & 0 & -1.931538 & -1.205736 & 1.148695 \\
\hline 19 & 8 & 0 & -4.693550 & -.627021 & -1.312390 \\
\hline 20 & 1 & 0 & 2.314592 & -3.792003 & .015484 \\
\hline 21 & 1 & 0 & 4.111739 & -2.132980 & -.550448 \\
\hline 22 & 1 & 0 & .030166 & -3.007145 & .522049 \\
\hline 23 & 1 & 0 & 5.065622 & 1.929692 & -.235713 \\
\hline 24 & 1 & 0 & 4.502029 & .957717 & 1.154353 \\
\hline 25 & 1 & 0 & 3.398073 & 2.138483 & .375701 \\
\hline 26 & 1 & 0 & -2.438202 & 2.639878 & .582453 \\
\hline 27 & 1 & 0 & -4.458515 & 1.557923 & -.030460 \\
\hline 28 & 1 & 0 & -2.956021 & -2.434598 & -.427673 \\
\hline 29 & 1 & 0 & -2.387801 & -.659269 & 1.989299 \\
\hline 30 & 1 & 0 & -1.549957 & -2.149261 & 1.549116 \\
\hline
\end{tabular}

\begin{tabular}{|c|c|c|c|c|}
\hline & & 1 & 2 & 3 \\
\hline & & A & A & A \\
\hline Frequencies & -- & 41.5043 & 60.1134 & 82.1901 \\
\hline Red. masses & -- & 6.9013 & 4.6347 & 2.1725 \\
\hline Frc consts & -- & .0070 & .0099 & .0086 \\
\hline IR Inten & -- & 1.8849 & 2.3228 & 4.8168 \\
\hline
\end{tabular}


Theoretical data for compound 13a :

Final Energy in Hartrees: HF $=-879.7447082(B 3 L Y P / 6-31++G(D, P))$

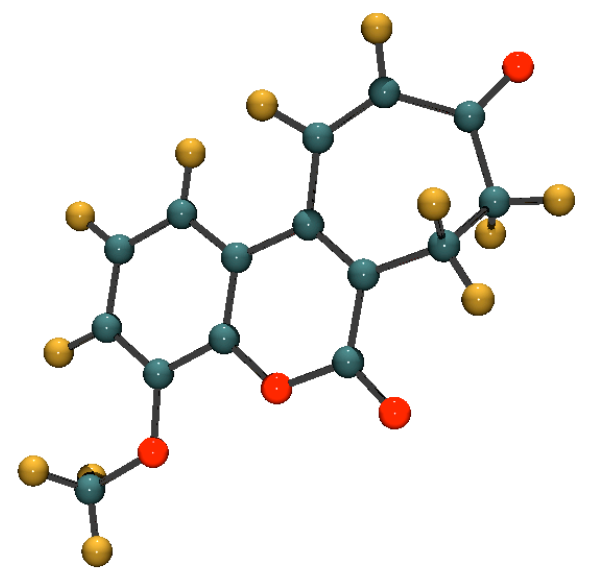

Standard orientation:

\begin{tabular}{|c|c|c|c|c|c|}
\hline \multirow{2}{*}{$\begin{array}{l}\text { Center } \\
\text { Number }\end{array}$} & \multirow{2}{*}{$\begin{array}{l}\text { Atomic } \\
\text { Number }\end{array}$} & \multirow{2}{*}{$\begin{array}{c}\text { Atomic } \\
\text { Type }\end{array}$} & \multicolumn{3}{|c|}{ Coordinates (Angstroms) } \\
\hline & & & $\mathrm{X}$ & $\mathrm{Y}$ & Z \\
\hline & 6 & 0 & $-2 \quad 313421$ & 2530857 & 059514 \\
\hline $\begin{array}{l}1 \\
2\end{array}$ & 6 & 0 & -3285576 & $\begin{array}{l}-2.530851 \\
-1.522069\end{array}$ & .059514 \\
\hline 2 & 0 & 0 & -3.285316 & -1.522069 & .151148 \\
\hline 3 & 6 & 0 & -2.907587 & -.181313 & .096624 \\
\hline 4 & 6 & 0 & -1.536649 & .129297 & -.059679 \\
\hline 5 & 6 & 0 & -.559558 & -.868676 & -.174515 \\
\hline 6 & 6 & 0 & -.973105 & -2.219105 & -.097839 \\
\hline 7 & 8 & 0 & -3.748446 & .878694 & .185674 \\
\hline 8 & 6 & 0 & -5.141028 & .626915 & .347323 \\
\hline 9 & 8 & 0 & -1.221952 & 1.454816 & -.086491 \\
\hline 10 & 6 & 0 & .075777 & 1.909311 & -.185967 \\
\hline 11 & 6 & 0 & 1.136599 & .899564 & -.326087 \\
\hline 12 & 6 & 0 & .831284 & -.437298 & -.341383 \\
\hline 13 & 8 & 0 & .253130 & 3.110256 & -.167064 \\
\hline 14 & 6 & 0 & 2.538257 & 1.432610 & -.462934 \\
\hline 15 & 6 & 0 & 3.495093 & .914964 & .624067 \\
\hline 16 & 6 & 0 & 4.013841 & -.489613 & .374859 \\
\hline 17 & 6 & 0 & 3.171645 & -1.490677 & -.331164 \\
\hline 18 & 6 & 0 & 1.846527 & -1.469861 & -.598601 \\
\hline 19 & 8 & 0 & 5.136678 & -.820998 & .737536 \\
\hline 20 & 1 & 0 & -5.611636 & 1.609062 & .393986 \\
\hline 21 & 1 & 0 & -5.341184 & .080480 & 1.277109 \\
\hline 22 & 1 & 0 & -5.545671 & .067202 & -.504807 \\
\hline 23 & 1 & 0 & 2.997377 & .923972 & 1.604415 \\
\hline 24 & 1 & 0 & 4.372134 & 1.562471 & .714992 \\
\hline 25 & 1 & 0 & 3.728810 & -2.385153 & -.599243 \\
\hline 26 & 1 & 0 & -2.622066 & -3.569685 & .119166 \\
\hline 27 & 1 & 0 & -.243862 & -3.018370 & -.141877 \\
\hline 28 & 1 & 0 & -4.327137 & -1.792752 & .272819 \\
\hline 29 & 1 & 0 & 2.490689 & 2.521517 & -.420468 \\
\hline 30 & 1 & 0 & 2.937119 & 1.167176 & -1.453275 \\
\hline 31 & 1 & 0 & 1.457562 & -2.367225 & -1.072250 \\
\hline
\end{tabular}

\begin{tabular}{|c|c|c|c|c|}
\hline & & 1 & 2 & 3 \\
\hline & & A & A & A \\
\hline Frequencies & -- & 31.8613 & 57.6338 & 67.2632 \\
\hline Red. masses & -- & 6.5382 & 4.7245 & 4.5207 \\
\hline Frc consts & -- & .0039 & .0092 & .0121 \\
\hline IR Inten & -- & 1.2865 & 10.3222 & .5703 \\
\hline
\end{tabular}


Theoretical data for compound $\mathbf{1 3 b}$ :

Final Energy in Hartrees: HF $=-879.7297326(B 3 L Y P / 6-31++G(D, P))$

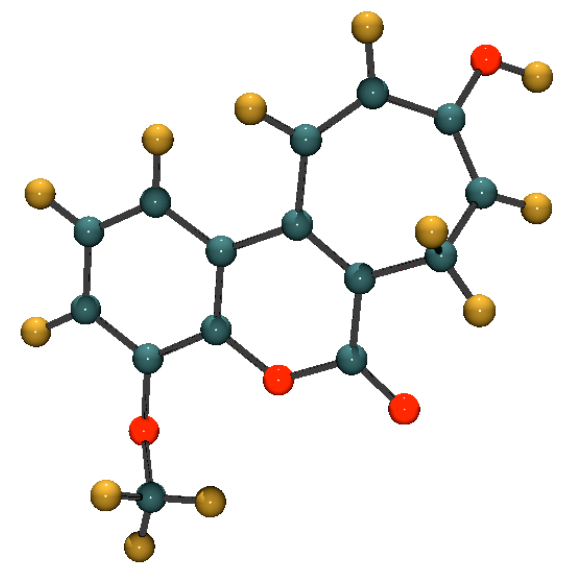

Standard orientation:

\begin{tabular}{|c|c|c|c|c|c|}
\hline \multirow{2}{*}{$\begin{array}{l}\text { Center } \\
\text { Number }\end{array}$} & \multirow{2}{*}{$\begin{array}{l}\text { Atomic } \\
\text { Number }\end{array}$} & \multirow{2}{*}{$\begin{array}{l}\text { Atomic } \\
\text { Type }\end{array}$} & \multicolumn{3}{|c|}{ Coordinates (Angstroms) } \\
\hline & & & $\mathrm{X}$ & $\mathrm{Y}$ & Z \\
\hline 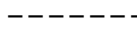 & & & & -- & --------- \\
\hline 1 & 6 & 0 & 2.248134 & 2.721570 & .047005 \\
\hline 2 & 6 & 0 & 3.232827 & 1.763404 & .315882 \\
\hline 3 & 6 & 0 & 2.913285 & .407731 & .338178 \\
\hline 4 & 6 & 0 & 1.580361 & .023099 & .087420 \\
\hline 5 & 6 & 0 & .582571 & .967972 & -.215226 \\
\hline 6 & 6 & 0 & .941194 & 2.332397 & -.216162 \\
\hline 7 & 8 & 0 & 3.883699 & -.488664 & .692121 \\
\hline 8 & 6 & 0 & 4.229686 & -1.508309 & -.261214 \\
\hline 9 & 8 & 0 & 1.300316 & -1.311232 & .179952 \\
\hline 10 & 6 & 0 & .034690 & -1.828936 & -.031864 \\
\hline 11 & 6 & 0 & -1.013467 & -.883574 & -.417937 \\
\hline 12 & 6 & 0 & -.769066 & .467197 & -.482597 \\
\hline 13 & 8 & 0 & -.104522 & -3.029056 & .095036 \\
\hline 14 & 6 & 0 & -2.361594 & -1.459645 & -.780408 \\
\hline 15 & 6 & 0 & -3.402190 & -1.027401 & .221718 \\
\hline 16 & 6 & 0 & -3.760053 & .270682 & .336952 \\
\hline 17 & 6 & 0 & -3.130832 & 1.352801 & -.403503 \\
\hline 18 & 6 & 0 & -1.831047 & 1.423356 & -.792789 \\
\hline 19 & 8 & 0 & -4.779326 & .723650 & 1.144955 \\
\hline 20 & 1 & 0 & 2.508494 & 3.775256 & .046406 \\
\hline 21 & 1 & 0 & 4.257577 & 2.049665 & .528494 \\
\hline 22 & 1 & 0 & .190204 & 3.089319 & -.405542 \\
\hline 23 & 1 & 0 & 5.084895 & -2.031332 & .168999 \\
\hline 24 & 1 & 0 & 4.521986 & -1.052304 & -1.215060 \\
\hline 25 & 1 & 0 & 3.406041 & -2.208979 & -.414248 \\
\hline 26 & 1 & 0 & -2.288795 & -2.546981 & -.805958 \\
\hline 27 & 1 & 0 & -2.638084 & -1.113113 & -1.786898 \\
\hline 28 & 1 & 0 & -3.883656 & -1.783489 & .839478 \\
\hline 29 & 1 & 0 & -3.764543 & 2.218699 & -.582316 \\
\hline 30 & 1 & 0 & -1.526733 & 2.338920 & -1.290520 \\
\hline 31 & 1 & 0 & -5.235721 & -.027320 & 1.547929 \\
\hline
\end{tabular}

\begin{tabular}{|c|c|c|c|c|}
\hline & & 1 & 2 & 3 \\
\hline & & A & A & A \\
\hline Frequencies & -- & 40.4855 & 56.5587 & 67.6228 \\
\hline Red. masses & -- & 5.8898 & 3.3867 & 2.1990 \\
\hline Frc consts & -- & .0057 & .0064 & .0059 \\
\hline IR Inten & -- & .6914 & 1.3699 & 3.4663 \\
\hline
\end{tabular}


Theoretical data for compound $13 \mathrm{c}$ :

Final Energy in Hartrees: HF $=-879.1767698$ (B3LYP / 6-31++G(D,P))

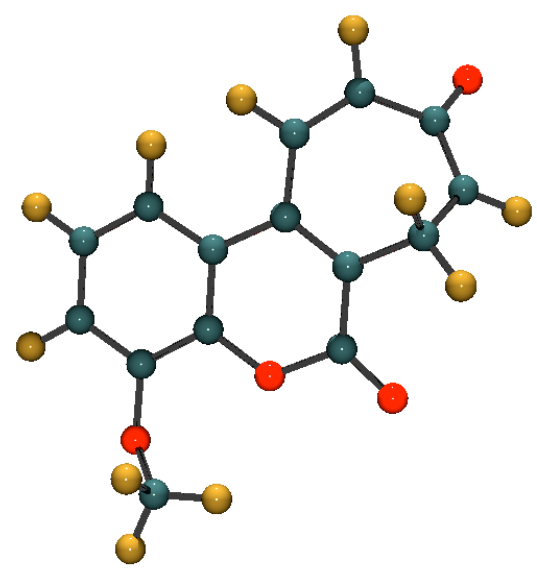

Standard orientation:

\begin{tabular}{|c|c|c|c|c|c|}
\hline \multirow{2}{*}{$\begin{array}{l}\text { Center } \\
\text { Number }\end{array}$} & \multirow{2}{*}{$\begin{array}{l}\text { Atomic } \\
\text { Number }\end{array}$} & \multirow{2}{*}{$\begin{array}{c}\text { Atomic } \\
\text { Type }\end{array}$} & \multicolumn{3}{|c|}{ Coordinates (Angstroms) } \\
\hline & & & $\mathrm{X}$ & $\mathrm{Y}$ & $\mathrm{Z}$ \\
\hline 1 & 6 & 0 & 2.258180 & 2.707007 & 0.020072 \\
\hline 2 & 6 & 0 & 3.231437 & 1.737316 & 0.291916 \\
\hline 3 & 6 & 0 & 2.876416 & 0.390133 & 0.334611 \\
\hline 4 & 6 & 0 & 1.537128 & 0.021285 & 0.103438 \\
\hline 5 & 6 & 0 & 0.549392 & 0.977626 & -0.210645 \\
\hline 6 & 6 & 0 & 0.941767 & 2.334915 & -0.226086 \\
\hline 7 & 8 & 0 & 3.844411 & -0.527165 & 0.682609 \\
\hline 8 & 6 & 0 & 4.130179 & -1.556921 & -0.271412 \\
\hline 9 & 8 & 0 & 1.237509 & -1.305211 & 0.212909 \\
\hline 10 & 6 & 0 & -0.049744 & -1.810920 & -0.021620 \\
\hline 11 & 6 & 0 & -1.071330 & -0.860311 & -0.421099 \\
\hline 12 & 6 & 0 & -0.806211 & 0.502121 & -0.485060 \\
\hline 13 & 8 & 0 & -0.165325 & -3.019487 & 0.091486 \\
\hline 14 & 6 & 0 & -2.416753 & -1.398903 & -0.834223 \\
\hline 15 & 6 & 0 & -3.422328 & -1.028501 & 0.223719 \\
\hline 16 & 6 & 0 & -3.838275 & 0.292863 & 0.449848 \\
\hline 17 & 6 & 0 & -3.170167 & 1.354633 & -0.350169 \\
\hline 18 & 6 & 0 & -1.866125 & 1.455685 & -0.753797 \\
\hline 19 & 8 & 0 & -4.755895 & 0.667790 & 1.255531 \\
\hline 20 & 1 & 0 & 2.533361 & 3.758052 & 0.006535 \\
\hline 21 & 1 & 0 & 4.264092 & 2.004643 & 0.492800 \\
\hline 22 & 1 & 0 & 0.198601 & 3.099770 & -0.415266 \\
\hline 23 & 1 & 0 & 4.977987 & -2.112324 & 0.136529 \\
\hline 24 & 1 & 0 & 4.415113 & -1.116069 & -1.236542 \\
\hline 25 & 1 & 0 & 3.277618 & -2.226949 & -0.404985 \\
\hline 26 & 1 & 0 & -2.350537 & -2.484499 & -0.932205 \\
\hline 27 & 1 & 0 & -2.669477 & -0.984370 & -1.825194 \\
\hline 28 & 1 & 0 & -3.873543 & -1.816649 & 0.822042 \\
\hline 29 & 1 & 0 & -3.793879 & 2.238733 & -0.485798 \\
\hline 30 & 1 & 0 & -1.566325 & 2.404077 & -1.195161 \\
\hline
\end{tabular}

\begin{tabular}{|c|c|c|c|c|}
\hline & & 1 & 2 & 3 \\
\hline & & A & A & A \\
\hline Frequencies & -- & 40.4683 & 54.8517 & 75.6483 \\
\hline Red. masses & -- & 6.5766 & 4.4953 & 2.0564 \\
\hline Frc consts & -- & .0063 & .0080 & .0069 \\
\hline IR Inten & -- & 1.6994 & .4552 & 3.7086 \\
\hline
\end{tabular}


Theoretical data for compound 12a :

Final Energy in Hartrees: HF $=-879.7161544(B 3 L Y P / 6-31++G(D, P))$

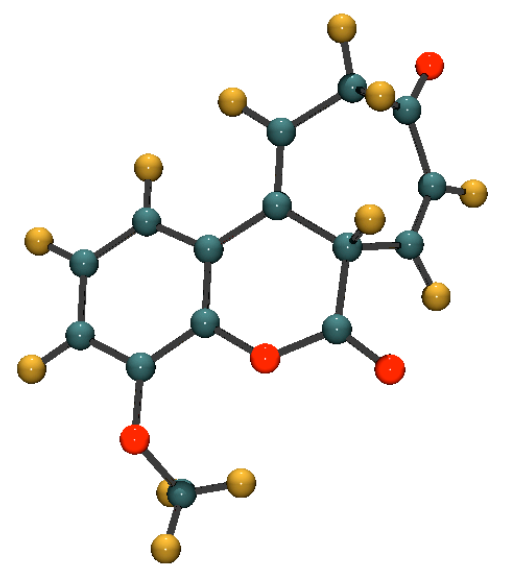

Standard orientation:

\begin{tabular}{|c|c|c|c|c|c|}
\hline \multirow{3}{*}{$\begin{array}{l}\text { Center } \\
\text { Number }\end{array}$} & \multirow{2}{*}{$\begin{array}{l}\text { Atomic } \\
\text { Number }\end{array}$} & \multirow{2}{*}{$\begin{array}{c}\text { Atomic } \\
\text { Type }\end{array}$} & \multicolumn{3}{|c|}{ Coordinates (Angstroms) } \\
\hline & & & $\mathrm{X}$ & $\mathrm{Y}$ & Z \\
\hline & 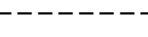 & & & -- & --------- \\
\hline 1 & 6 & 0 & 2.157522 & 2.747102 & .304289 \\
\hline 2 & 6 & 0 & 3.192667 & 1.809808 & .232235 \\
\hline 3 & 6 & 0 & 2.906108 & .469345 & -.022237 \\
\hline 4 & 6 & 0 & 1.566299 & .088695 & -.221535 \\
\hline 5 & 6 & 0 & .513486 & 1.012111 & -.170525 \\
\hline 6 & 6 & 0 & .838087 & 2.352963 & .115694 \\
\hline 7 & 8 & 0 & 3.940772 & -.416923 & -.154972 \\
\hline 8 & 6 & 0 & 4.049180 & -1.450060 & .838862 \\
\hline 9 & 8 & 0 & 1.367028 & -1.258275 & -.497477 \\
\hline 10 & 6 & 0 & .140743 & -1.842796 & -.471446 \\
\hline 11 & 6 & 0 & -1.103106 & -.958751 & -.455747 \\
\hline 12 & 6 & 0 & -.859600 & .549213 & -.431108 \\
\hline 13 & 8 & 0 & .073917 & -3.047539 & -.503295 \\
\hline 14 & 6 & 0 & -2.057941 & -1.461385 & .617351 \\
\hline 15 & 6 & 0 & -3.242224 & -.925692 & .962084 \\
\hline 16 & 6 & 0 & -3.917847 & .243304 & .349868 \\
\hline 17 & 6 & 0 & -3.307689 & .880705 & -.900324 \\
\hline 18 & 6 & 0 & -1.899120 & 1.368021 & -.672412 \\
\hline 19 & 8 & 0 & -4.964042 & .666374 & .822475 \\
\hline 20 & 1 & 0 & -1.569801 & -1.198179 & -1.427171 \\
\hline 21 & 1 & 0 & 4.967708 & -1.989312 & .603310 \\
\hline 22 & 1 & 0 & 3.199584 & -2.136317 & .795824 \\
\hline 23 & 1 & 0 & 4.129897 & -1.008294 & 1.839577 \\
\hline 24 & 1 & 0 & -3.808846 & -1.380906 & 1.770604 \\
\hline 25 & 1 & 0 & 4.229957 & 2.095412 & .371394 \\
\hline 26 & 1 & 0 & -3.965978 & 1.695855 & -1.204408 \\
\hline 27 & 1 & 0 & -3.325635 & .122272 & -1.700434 \\
\hline 28 & 1 & 0 & -1.752655 & 2.443225 & -.684839 \\
\hline 29 & 1 & 0 & 2.384498 & 3.786451 & .519588 \\
\hline 30 & 1 & 0 & .045851 & 3.088026 & .199879 \\
\hline 31 & 1 & 0 & -1.735367 & -2.362810 & 1.132520 \\
\hline
\end{tabular}

\begin{tabular}{|c|c|c|c|c|}
\hline & & 1 & 2 & 3 \\
\hline & & A & A & A \\
\hline Frequencies & -- & 34.8250 & 54.7312 & 61.2133 \\
\hline Red. masses & -- & 6.6370 & 3.9926 & 5.0008 \\
\hline Frc consts & -- & .0047 & .0070 & .0110 \\
\hline IR Inten & -- & .5916 & 3.8859 & 3.0258 \\
\hline
\end{tabular}


Theoretical data for compound $\mathbf{1 2 b}$ :

Final Energy in Hartrees: HF $=-879.7154875(B 3 L Y P / 6-31++G(D, P))$

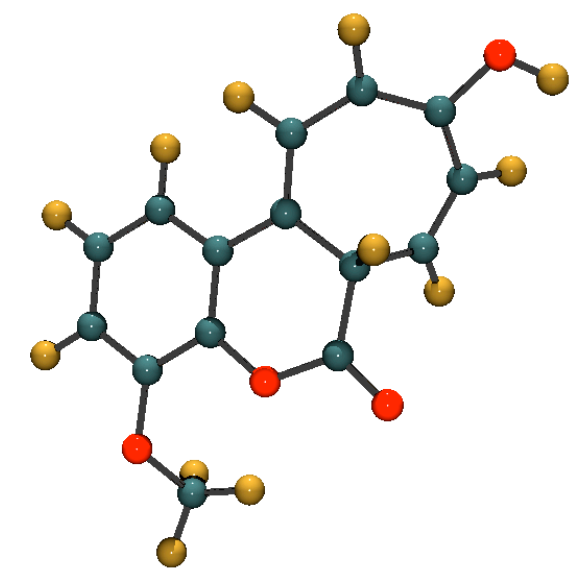

Standard orientation:

\begin{tabular}{|c|c|c|c|c|c|}
\hline \multirow{2}{*}{$\begin{array}{l}\text { Center } \\
\text { Number }\end{array}$} & \multirow{2}{*}{$\begin{array}{l}\text { Atomic } \\
\text { Number }\end{array}$} & \multirow{2}{*}{$\begin{array}{c}\text { Atomic } \\
\text { Type }\end{array}$} & \multicolumn{3}{|c|}{ Coordinates (Angstroms) } \\
\hline & & & $\mathrm{X}$ & $\mathrm{Y}$ & Z \\
\hline 1 & 6 & 0 & 2.355393 & 2.675332 & .304424 \\
\hline 2 & 6 & 0 & 3.330982 & 1.682857 & .169081 \\
\hline 3 & 6 & 0 & 2.957362 & .361762 & -.080352 \\
\hline 4 & 6 & 0 & 1.591801 & .055642 & -.214580 \\
\hline 5 & 6 & 0 & .594053 & 1.037413 & -.093902 \\
\hline 6 & 6 & 0 & 1.007427 & 2.355002 & .190512 \\
\hline 7 & 8 & 0 & 3.938588 & -.574831 & -.272336 \\
\hline 8 & 6 & 0 & 4.024765 & -1.639471 & .688602 \\
\hline 9 & 8 & 0 & 1.302453 & -1.272899 & -.495412 \\
\hline 10 & 6 & 0 & .036340 & -1.780125 & -.479620 \\
\hline 11 & 6 & 0 & -1.136400 & -.828857 & -.325685 \\
\hline 12 & 6 & 0 & -.803824 & .649941 & -.288121 \\
\hline 13 & 8 & 0 & -.104210 & -2.972799 & -.602175 \\
\hline 14 & 6 & 0 & -1.948359 & -1.164472 & .903202 \\
\hline 15 & 6 & 0 & -3.214621 & -.715932 & 1.041459 \\
\hline 16 & 6 & 0 & -3.856810 & .209998 & .132292 \\
\hline 17 & 6 & 0 & -3.224435 & 1.220778 & -.547366 \\
\hline 18 & 6 & 0 & -1.824927 & 1.533098 & -.500044 \\
\hline 19 & 8 & 0 & -5.230750 & .197458 & .072648 \\
\hline 20 & 1 & 0 & 2.651848 & 3.697912 & .516212 \\
\hline 21 & 1 & 0 & 4.388609 & 1.908175 & .256270 \\
\hline 22 & 1 & 0 & .258887 & 3.126335 & .333113 \\
\hline 23 & 1 & 0 & 4.911672 & -2.212559 & .414156 \\
\hline 24 & 1 & 0 & 3.143847 & -2.285175 & .649820 \\
\hline 25 & 1 & 0 & 4.151239 & -1.231337 & 1.699081 \\
\hline 26 & 1 & 0 & -1.775744 & -1.015828 & -1.202860 \\
\hline 27 & 1 & 0 & -1.496203 & -1.770462 & 1.683527 \\
\hline 28 & 1 & 0 & -3.791788 & -1.025441 & 1.912186 \\
\hline 29 & 1 & 0 & -3.877518 & 1.925239 & -1.056915 \\
\hline 30 & 1 & 0 & -1.575588 & 2.577962 & -.667630 \\
\hline 31 & 1 & 0 & -5.570374 & -.628041 & .441821 \\
\hline
\end{tabular}

\begin{tabular}{|c|c|c|c|c|}
\hline & & 1 & 2 & 3 \\
\hline & & A & A & A \\
\hline Frequencies & -- & 35.8372 & 58.2511 & 74.5111 \\
\hline Red. masses & -- & 6.3175 & 2.8679 & 3.0436 \\
\hline Frc consts & -- & .0048 & .0057 & .0100 \\
\hline IR Inten & -- & 1.1746 & .9009 & 2.3515 \\
\hline
\end{tabular}


Theoretical data for compound 14a :

Final Energy in Hartrees: HF $=-879.7186584(B 3 L Y P / 6-31++G(D, P))$

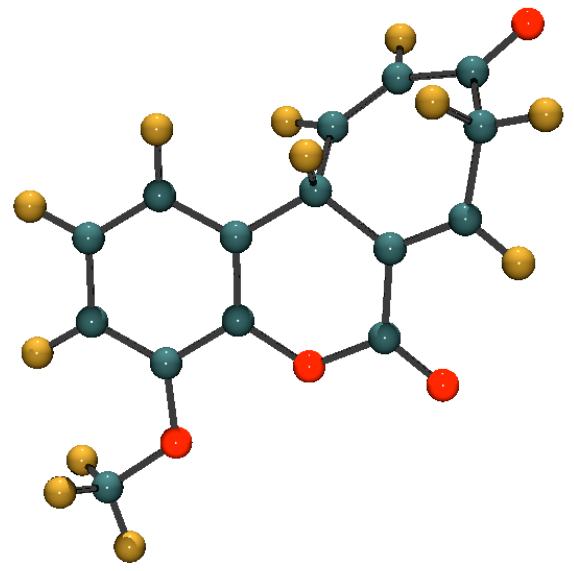

Standard orientation:

\begin{tabular}{|c|c|c|c|c|c|}
\hline \multirow{2}{*}{$\begin{array}{l}\text { Center } \\
\text { Number }\end{array}$} & \multirow{2}{*}{$\begin{array}{l}\text { Atomic } \\
\text { Number }\end{array}$} & \multirow{2}{*}{$\begin{array}{l}\text { Atomic } \\
\text { Type }\end{array}$} & \multicolumn{3}{|c|}{ Coordinates (Angstroms) } \\
\hline & & & $\mathrm{X}$ & $\mathrm{Y}$ & Z \\
\hline 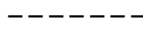 & & & 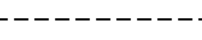 & $=-$ & --------- \\
\hline 1 & 6 & 0 & -2.428058 & -2.456413 & -.439943 \\
\hline 2 & 6 & 0 & -3.335544 & -1.427260 & -.158211 \\
\hline 3 & 6 & 0 & -2.873763 & -.125515 & .049658 \\
\hline 4 & 6 & 0 & -1.483439 & .126354 & -.024507 \\
\hline 5 & 6 & 0 & -.579180 & -.894124 & -.294561 \\
\hline 6 & 6 & 0 & -1.065417 & -2.194729 & -.509551 \\
\hline 7 & 8 & 0 & -3.657574 & .947661 & .323635 \\
\hline 8 & 6 & 0 & -5.065721 & .755571 & .408334 \\
\hline 9 & 8 & 0 & -1.109903 & 1.439257 & .192651 \\
\hline 10 & 6 & 0 & .167082 & 1.892607 & -.006218 \\
\hline 11 & 6 & 0 & 1.211134 & .891339 & -.353420 \\
\hline 12 & 6 & 0 & .905001 & -.600395 & -.362595 \\
\hline 13 & 8 & 0 & .372206 & 3.077921 & .125298 \\
\hline 14 & 6 & 0 & 2.447623 & 1.337071 & -.629574 \\
\hline 15 & 6 & 0 & 3.598602 & .420356 & -.935892 \\
\hline 16 & 6 & 0 & 4.019127 & -.453416 & .254711 \\
\hline 17 & 6 & 0 & 3.006911 & -1.271852 & .962699 \\
\hline 18 & 6 & 0 & 1.686197 & -1.347735 & .712463 \\
\hline 19 & 8 & 0 & 5.191367 & -.494386 & .600906 \\
\hline 20 & 1 & 0 & 1.259157 & -.989829 & -1.332635 \\
\hline 21 & 1 & 0 & -5.481221 & 1.737634 & .635133 \\
\hline 22 & 1 & 0 & -5.324933 & .054488 & 1.211217 \\
\hline 23 & 1 & 0 & -5.476791 & .395216 & -.542765 \\
\hline 24 & 1 & 0 & 3.425874 & -1.863149 & 1.773218 \\
\hline 25 & 1 & 0 & 4.482129 & .973159 & -1.257628 \\
\hline 26 & 1 & 0 & 3.317828 & -.268174 & -1.750519 \\
\hline 27 & 1 & 0 & 2.630032 & 2.407375 & -.599854 \\
\hline 28 & 1 & 0 & -4.395096 & -1.645595 & -.108062 \\
\hline 29 & 1 & 0 & -2.798814 & -3.462735 & -.607626 \\
\hline 30 & 1 & 0 & -.365599 & -2.994784 & -.734698 \\
\hline 31 & 1 & 0 & 1.095335 & -2.018382 & 1.333952 \\
\hline
\end{tabular}

\begin{tabular}{|c|c|c|c|c|}
\hline & & 1 & 2 & 3 \\
\hline & & A & A & A \\
\hline Frequencies & -- & 37.1078 & 61.5411 & 64.4137 \\
\hline Red. masses & -- & 5.8085 & 6.9639 & 4.5436 \\
\hline Frc consts & -- & .0047 & .0155 & .0111 \\
\hline IR Inten & -- & .4599 & 6.3032 & .4052 \\
\hline
\end{tabular}


Theoretical data for compound $\mathbf{1 4 b}$ :

Final Energy in Hartrees: HF $=-879.7203451(B 3 L Y P / 6-31++G(D, P))$

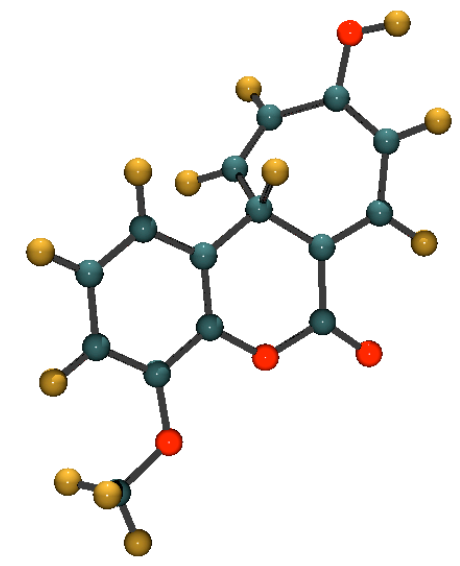

Standard orientation:

\begin{tabular}{|c|c|c|c|c|c|}
\hline \multirow{2}{*}{$\begin{array}{l}\text { Center } \\
\text { Number }\end{array}$} & \multirow{2}{*}{$\begin{array}{l}\text { Atomic } \\
\text { Number }\end{array}$} & \multirow{2}{*}{$\begin{array}{c}\text { Atomic } \\
\text { Type }\end{array}$} & \multicolumn{3}{|c|}{ Coordinates (Angstroms) } \\
\hline & & & $\mathrm{X}$ & $\mathrm{Y}$ & Z \\
\hline 1 & 6 & 0 & -2.302087 & -2.519815 & -.434298 \\
\hline 2 & 6 & 0 & -3.267516 & -1.530642 & -.205651 \\
\hline 3 & 6 & 0 & -2.876941 & -.204986 & -.000965 \\
\hline 4 & 6 & 0 & -1.498893 & .113357 & -.025110 \\
\hline 5 & 6 & 0 & -.538042 & -.867962 & -.244336 \\
\hline 6 & 6 & 0 & -.951528 & -2.193519 & -.455033 \\
\hline 7 & 8 & 0 & -3.724581 & .832651 & .224377 \\
\hline 8 & 6 & 0 & -5.122593 & .570978 & .249799 \\
\hline 9 & 8 & 0 & -1.193800 & 1.444316 & .183881 \\
\hline 10 & 6 & 0 & .075745 & 1.949155 & .028450 \\
\hline 11 & 6 & 0 & 1.168273 & .988245 & -.225547 \\
\hline 12 & 6 & 0 & .927832 & -.506104 & -.239028 \\
\hline 13 & 8 & 0 & .211671 & 3.151788 & .109336 \\
\hline 14 & 6 & 0 & 2.410888 & 1.485001 & -.488270 \\
\hline 15 & 6 & 0 & 3.618654 & .723579 & -.604243 \\
\hline 16 & 6 & 0 & 3.897724 & -.447497 & .062072 \\
\hline 17 & 6 & 0 & 3.021944 & -1.111704 & 1.005160 \\
\hline 18 & 6 & 0 & 1.673840 & -1.090359 & .943216 \\
\hline 19 & 8 & 0 & 5.142422 & -1.011227 & .019009 \\
\hline 20 & 1 & 0 & -2.618586 & -3.545216 & -.598372 \\
\hline 21 & 1 & 0 & -4.316721 & -1.799204 & -.193540 \\
\hline 22 & 1 & 0 & -.202908 & -2.960084 & -.634677 \\
\hline 23 & 1 & 0 & -5.597285 & 1.534260 & .438612 \\
\hline 24 & 1 & 0 & -5.383890 & -.128761 & 1.053513 \\
\hline 25 & 1 & 0 & -5.471973 & .173316 & -.711268 \\
\hline 26 & 1 & 0 & 1.396544 & -.906992 & -1.152292 \\
\hline 27 & 1 & 0 & 2.494262 & 2.562413 & -.608988 \\
\hline 28 & 1 & 0 & 4.443738 & 1.199189 & -1.134619 \\
\hline 29 & 1 & 0 & 3.521378 & -1.626693 & 1.821728 \\
\hline 30 & 1 & 0 & 1.091573 & -1.511459 & 1.759483 \\
\hline 31 & 1 & 0 & 5.734387 & -.477373 & -.529699 \\
\hline
\end{tabular}

\begin{tabular}{|c|c|c|c|c|}
\hline & & 1 & 2 & 3 \\
\hline & & A & A & A \\
\hline Frequencies & -- & 39.4168 & 64.6039 & 85.0857 \\
\hline Red. masses & -- & 4.9099 & 4.5417 & 4.3780 \\
\hline Frc consts & -- & .0045 & .0112 & .0187 \\
\hline IR Inten & -- & 1.8318 & 2.3416 & 1.1459 \\
\hline
\end{tabular}


Theoretical data for compound 14c :

Final Energy in Hartrees: HF $=-879.1933354(B 3 L Y P / 6-31++G(D, P))$

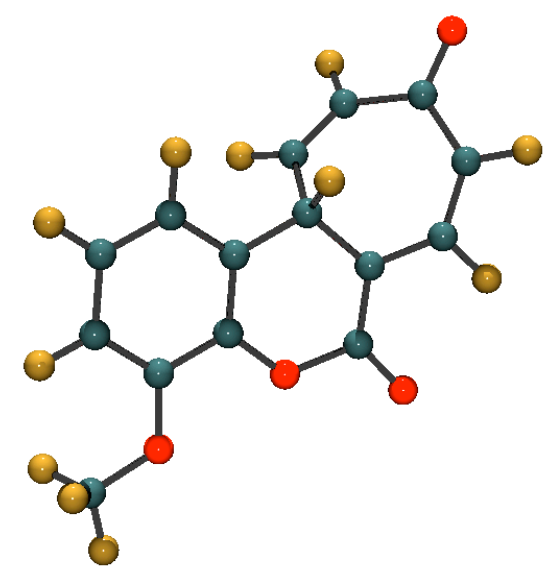

Standard orientation:

\begin{tabular}{|c|c|c|c|c|c|}
\hline \multirow{2}{*}{$\begin{array}{l}\text { Center } \\
\text { Number }\end{array}$} & \multirow{2}{*}{$\begin{array}{l}\text { Atomic } \\
\text { Number }\end{array}$} & \multirow{2}{*}{$\begin{array}{c}\text { Atomic } \\
\text { Type }\end{array}$} & \multicolumn{3}{|c|}{ Coordinates (Angstroms) } \\
\hline & & & $\mathrm{X}$ & Y & Z \\
\hline & 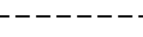 & & & & --------- \\
\hline 1 & 6 & 0 & -2.256912 & -2.517871 & -.424628 \\
\hline 2 & 6 & 0 & -3.229952 & -1.530435 & -.206917 \\
\hline 3 & 6 & 0 & -2.841695 & -.205508 & -.004271 \\
\hline 4 & 6 & 0 & -1.465666 & .136787 & -.016755 \\
\hline 5 & 6 & 0 & -.498777 & -.848630 & -.223588 \\
\hline 6 & 6 & 0 & -.910031 & -2.176345 & -.432213 \\
\hline 7 & 8 & 0 & -3.711672 & .831001 & .213157 \\
\hline 8 & 6 & 0 & -5.095874 & .546112 & .224565 \\
\hline 9 & 8 & 0 & -1.173144 & 1.451728 & .184275 \\
\hline 10 & 6 & 0 & .151900 & 1.958763 & .035699 \\
\hline 11 & 6 & 0 & 1.201216 & 1.012257 & -.166304 \\
\hline 12 & 6 & 0 & .967110 & -.482513 & -.219164 \\
\hline 13 & 8 & 0 & .224094 & 3.181355 & .097216 \\
\hline 14 & 6 & 0 & 2.491843 & 1.486971 & -.437165 \\
\hline 15 & 6 & 0 & 3.682689 & .767606 & -.539034 \\
\hline 16 & 6 & 0 & 4.022863 & -.516361 & .027532 \\
\hline 17 & 6 & 0 & 3.072434 & -1.184897 & .963098 \\
\hline 18 & 6 & 0 & 1.729359 & -1.165589 & .903116 \\
\hline 19 & 8 & 0 & 5.165646 & -1.023801 & -.107814 \\
\hline 20 & 1 & 0 & -2.564965 & -3.547179 & -.586924 \\
\hline 21 & 1 & 0 & -4.278877 & -1.803509 & -.201590 \\
\hline 22 & 1 & 0 & -.151311 & -2.936975 & -.597251 \\
\hline 23 & 1 & 0 & -5.594895 & 1.500216 & .406185 \\
\hline 24 & 1 & 0 & -5.360708 & -.159042 & 1.025354 \\
\hline 25 & 1 & 0 & -5.435418 & .137060 & -.737713 \\
\hline 26 & 1 & 0 & 1.413699 & -.846133 & -1.163969 \\
\hline 27 & 1 & 0 & 2.554611 & 2.558221 & -.625899 \\
\hline 28 & 1 & 0 & 4.542887 & 1.282882 & -.963156 \\
\hline 29 & 1 & 0 & 3.566209 & -1.737341 & 1.761090 \\
\hline 30 & 1 & 0 & 1.146338 & -1.652549 & 1.685374 \\
\hline
\end{tabular}

\begin{tabular}{|c|c|c|c|c|}
\hline & & 1 & 2 & 3 \\
\hline & & A & A & A \\
\hline Frequencies & -- & 39.7482 & 61.4077 & 76.6007 \\
\hline Red. masses & -- & 4.7705 & 4.6043 & 5.4395 \\
\hline Frc consts & -- & .0044 & .0102 & .0188 \\
\hline IR Inten & -- & .1498 & 1.2772 & 7.0362 \\
\hline
\end{tabular}




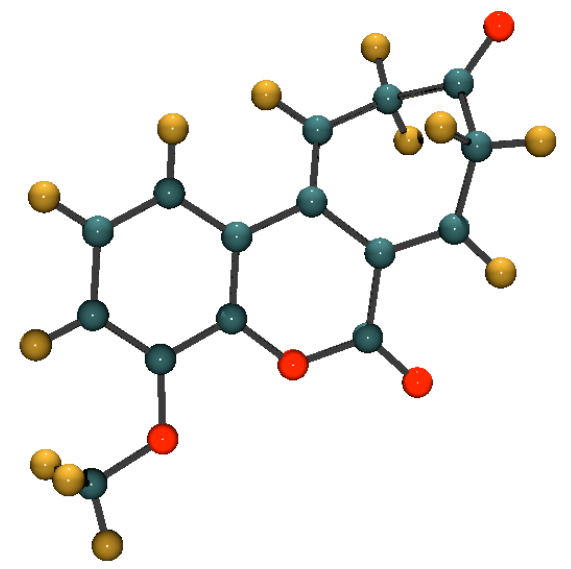

Standard orientation:

\begin{tabular}{|c|c|c|c|c|c|}
\hline \multirow{2}{*}{$\begin{array}{l}\text { Center } \\
\text { Number }\end{array}$} & \multirow{2}{*}{$\begin{array}{l}\text { Atomic } \\
\text { Number }\end{array}$} & \multirow{2}{*}{$\begin{array}{c}\text { Atomic } \\
\text { Type }\end{array}$} & \multicolumn{3}{|c|}{ Coordinates (Angstroms) } \\
\hline & & & $\mathrm{X}$ & $\mathrm{Y}$ & Z \\
\hline & & & & & 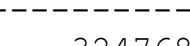 \\
\hline 1 & 6 & 0 & 2.311009 & -2.527939 & .324768 \\
\hline 2 & 6 & 0 & 3.281105 & -1.518780 & .251171 \\
\hline 3 & 6 & 0 & 2.895084 & -.192142 & .043963 \\
\hline 4 & 6 & 0 & 1.520691 & .101334 & -.091232 \\
\hline 5 & 6 & 0 & .549101 & -.902342 & -.036371 \\
\hline 6 & 6 & 0 & .962739 & -2.229983 & .185741 \\
\hline 7 & 8 & 0 & 3.742321 & .865232 & -.043142 \\
\hline 8 & 6 & 0 & 5.139882 & .625065 & .080462 \\
\hline 9 & 8 & 0 & 1.212119 & 1.429041 & -.322150 \\
\hline 10 & 6 & 0 & -.064550 & 1.918322 & -.213411 \\
\hline 11 & 6 & 0 & -1.136157 & .920373 & .065785 \\
\hline 12 & 6 & 0 & -.856974 & -.493196 & -.220746 \\
\hline 13 & 8 & 0 & -.236746 & 3.108099 & -.344168 \\
\hline 14 & 6 & 0 & -2.291527 & 1.372462 & .610468 \\
\hline 15 & 6 & 0 & -3.394678 & .498049 & 1.119018 \\
\hline 16 & 6 & 0 & -4.095316 & -.401148 & .087301 \\
\hline 17 & 6 & 0 & -3.213951 & -.895691 & -1.062952 \\
\hline 18 & 6 & 0 & -1.821990 & -1.321696 & -.693894 \\
\hline 19 & 8 & 0 & -5.264521 & -.696550 & .189425 \\
\hline 20 & 1 & 0 & 5.614512 & 1.600938 & -.024627 \\
\hline 21 & 1 & 0 & 5.500787 & -.044179 & -.710296 \\
\hline 22 & 1 & 0 & 5.386654 & .202343 & 1.062216 \\
\hline 23 & 1 & 0 & -4.161394 & 1.070634 & 1.643938 \\
\hline 24 & 1 & 0 & -2.971324 & -.224324 & 1.837361 \\
\hline 25 & 1 & 0 & -2.394614 & 2.446478 & .740402 \\
\hline 26 & 1 & 0 & -3.157621 & -.026904 & -1.741269 \\
\hline 27 & 1 & 0 & -3.753540 & -1.688865 & -1.583330 \\
\hline 28 & 1 & 0 & -1.567796 & -2.357364 & -.900217 \\
\hline 29 & 1 & 0 & 2.623756 & -3.552154 & .502580 \\
\hline 30 & 1 & 0 & .220555 & -3.016380 & .269789 \\
\hline 31 & 1 & 0 & 4.327838 & -1.772926 & .363310 \\
\hline
\end{tabular}

\begin{tabular}{lrrr} 
& \multicolumn{1}{c}{1} & \multicolumn{1}{c}{2} & \multicolumn{1}{c}{3} \\
& & \multicolumn{1}{c}{ A } & \multicolumn{1}{c}{ A } \\
Frequencies -- & 37.3845 & 49.5040 & 76.5492 \\
Red. masses -- & 6.7381 & 5.4742 & 4.4583 \\
Frc consts -- & .0055 & .0079 & .0154 \\
IR Inten -- & 1.3950 & 4.8171 & 1.1114
\end{tabular}


Theoretical data for compound $\mathbf{1 5 b}$ :

Final Energy in Hartrees: HF $=-879.7291446(B 3 L Y P / 6-31++G(D, P))$

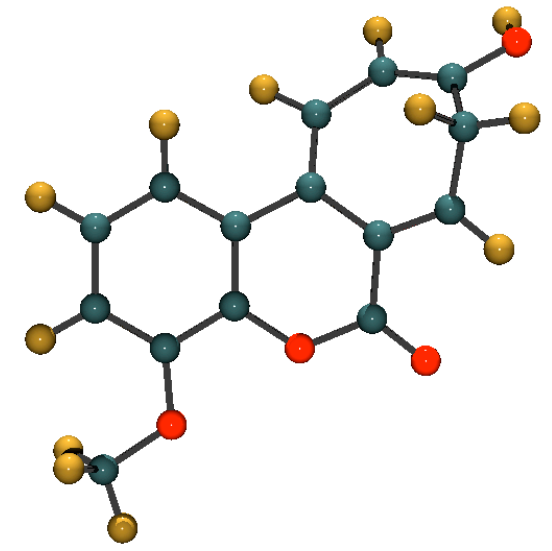

Standard orientation:

\begin{tabular}{|c|c|c|c|c|c|}
\hline \multirow{2}{*}{$\begin{array}{l}\text { Center } \\
\text { Number }\end{array}$} & \multirow{2}{*}{$\begin{array}{l}\text { Atomic } \\
\text { Number }\end{array}$} & \multirow{2}{*}{$\begin{array}{l}\text { Atomic } \\
\text { Type }\end{array}$} & \multicolumn{3}{|c|}{ Coordinates (Angstroms) } \\
\hline & & & $\mathrm{X}$ & $\mathrm{Y}$ & Z \\
\hline 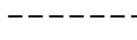 & & & & --1 & --------- \\
\hline 1 & 6 & 0 & 2.452177 & -2.471743 & .301562 \\
\hline 2 & 6 & 0 & 3.376013 & -1.425507 & .170925 \\
\hline 3 & 6 & 0 & 2.922618 & -.116454 & -.009047 \\
\hline 4 & 6 & 0 & 1.532521 & .120608 & -.063124 \\
\hline 5 & 6 & 0 & .602907 & -.918013 & .049285 \\
\hline 6 & 6 & 0 & 1.087206 & -2.227568 & .247458 \\
\hline 7 & 8 & 0 & 3.719796 & .976315 & -.143208 \\
\hline 8 & 6 & 0 & 5.129619 & .793586 & -.096146 \\
\hline 9 & 8 & 0 & 1.156693 & 1.431383 & -.271378 \\
\hline 10 & 6 & 0 & -.139784 & 1.857279 & -.141651 \\
\hline 11 & 6 & 0 & -1.163184 & .823822 & .190349 \\
\hline 12 & 6 & 0 & -.827234 & -.576796 & -.033439 \\
\hline 13 & 8 & 0 & -.367624 & 3.036014 & -.296596 \\
\hline 14 & 6 & 0 & -2.368943 & 1.276494 & .624110 \\
\hline 15 & 6 & 0 & -3.428831 & .352932 & 1.150660 \\
\hline 16 & 6 & 0 & -3.937662 & -.412519 & -.036760 \\
\hline 17 & 6 & 0 & -3.155154 & -1.306813 & -.705647 \\
\hline 18 & 6 & 0 & -1.752794 & -1.496706 & -.482072 \\
\hline 19 & 8 & 0 & -5.238088 & -.159078 & -.343281 \\
\hline 20 & 1 & 0 & 5.558258 & 1.788282 & -.221923 \\
\hline 21 & 1 & 0 & 5.475320 & .141417 & -.907981 \\
\hline 22 & 1 & 0 & 5.446701 & .378378 & .868685 \\
\hline 23 & 1 & 0 & -3.624222 & -1.949613 & -1.449853 \\
\hline 24 & 1 & 0 & -4.247186 & .902722 & 1.618551 \\
\hline 25 & 1 & 0 & -3.001893 & -.346426 & 1.882264 \\
\hline 26 & 1 & 0 & -2.560444 & 2.345003 & .591755 \\
\hline 27 & 1 & 0 & -1.359379 & -2.453114 & -.817727 \\
\hline 28 & 1 & 0 & 2.814963 & -3.482589 & .460764 \\
\hline 29 & 1 & 0 & .385244 & -3.042283 & .387485 \\
\hline 30 & 1 & 0 & 4.437019 & -1.636710 & .220276 \\
\hline 31 & 1 & 0 & -5.467440 & -.557752 & -1.195382 \\
\hline
\end{tabular}

\begin{tabular}{|c|c|c|c|c|}
\hline & & 1 & 2 & 3 \\
\hline & & A & A & A \\
\hline Frequencies & -- & 40.5628 & 78.1995 & 86.3837 \\
\hline Red. masses & -- & 4.9080 & 4.5149 & 4.1037 \\
\hline Frc consts & -- & .0048 & .0163 & .0180 \\
\hline IR Inten & -- & 2.9038 & .5360 & 1.7504 \\
\hline
\end{tabular}


Theoretical data for compound $15 \mathrm{c}$ :

Final Energy in Hartrees: HF $=-879.2055479(B 3 L Y P / 6-31++G(D, P))$

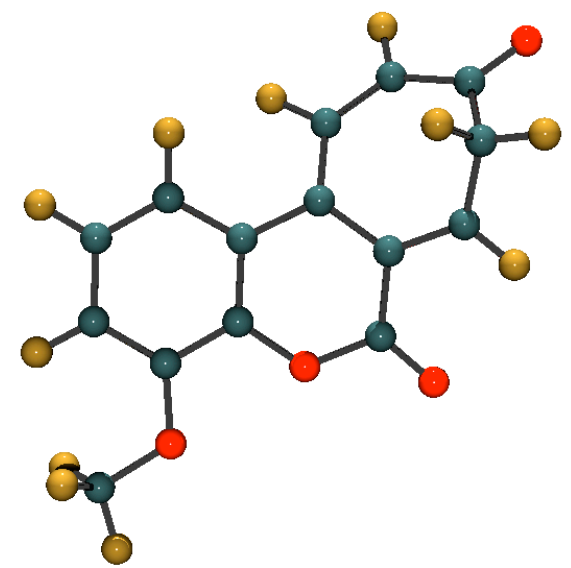

Standard orientation:

\begin{tabular}{|c|c|c|c|c|c|}
\hline \multirow{2}{*}{$\begin{array}{l}\text { Center } \\
\text { Number }\end{array}$} & \multirow{2}{*}{$\begin{array}{l}\text { Atomic } \\
\text { Number }\end{array}$} & \multirow{2}{*}{$\begin{array}{c}\text { Atomic } \\
\text { Type }\end{array}$} & \multicolumn{3}{|c|}{ Coordinates (Angstroms) } \\
\hline & & & $\mathrm{X}$ & $Y$ & Z \\
\hline & & & --------- & ---------- & ---------- \\
\hline 1 & 6 & 0 & 2.390492 & -2.488787 & .253725 \\
\hline 2 & 6 & 0 & 3.337309 & -1.455913 & .166428 \\
\hline 3 & 6 & 0 & 2.888422 & -.138110 & .019958 \\
\hline 4 & 6 & 0 & 1.508911 & .123409 & -.047024 \\
\hline 5 & 6 & 0 & .544693 & -.899304 & .039652 \\
\hline 6 & 6 & 0 & 1.029889 & -2.222583 & .201784 \\
\hline 7 & 8 & 0 & 3.715588 & .953953 & -.081024 \\
\hline 8 & 6 & 0 & 5.111041 & .733664 & -.041981 \\
\hline 9 & 8 & 0 & 1.151601 & 1.439179 & -.248596 \\
\hline 10 & 6 & 0 & -.156242 & 1.877657 & -.168009 \\
\hline 11 & 6 & 0 & -1.192217 & .867665 & .138319 \\
\hline 12 & 6 & 0 & -.861061 & -.536695 & -.034373 \\
\hline 13 & 8 & 0 & -.336711 & 3.068720 & -.345286 \\
\hline 14 & 6 & 0 & -2.425266 & 1.333980 & .502878 \\
\hline 15 & 6 & 0 & -3.490545 & .449728 & 1.067919 \\
\hline 16 & 6 & 0 & -4.097018 & -.418521 & -.051232 \\
\hline 17 & 6 & 0 & -3.198306 & -1.358384 & -.659633 \\
\hline 18 & 6 & 0 & -1.821369 & -1.475951 & -.466997 \\
\hline 19 & 8 & 0 & -5.309909 & -.321441 & -.329933 \\
\hline 20 & 1 & 0 & 5.571413 & 1.718727 & -.145046 \\
\hline 21 & 1 & 0 & 5.445055 & .089451 & -.867620 \\
\hline 22 & 1 & 0 & 5.426867 & .284869 & .910499 \\
\hline 23 & 1 & 0 & -3.688222 & -2.134703 & -1.244825 \\
\hline 24 & 1 & 0 & -4.299193 & 1.032836 & 1.515533 \\
\hline 25 & 1 & 0 & -3.058480 & -.213151 & 1.834018 \\
\hline 26 & 1 & 0 & -2.622078 & 2.396647 & .398796 \\
\hline 27 & 1 & 0 & -1.400211 & -2.421288 & -.812415 \\
\hline 28 & 1 & 0 & 2.734202 & -3.512162 & .383788 \\
\hline 29 & 1 & 0 & .318616 & -3.033377 & .316397 \\
\hline 30 & 1 & 0 & 4.395101 & -1.682254 & .221121 \\
\hline
\end{tabular}

1

A

Frequencies -

Red. masses --

Frc consts --

IR Inten
39.8844

4.6962

.0044

.4875
2

77.0514

4.2661

.0149

3.6547
3

A

81.3043

4.4123

.0172

.9263 
Theoretical data for compound $\mathbf{6 b}$ :

Final Energy in Hartrees: HF $=-879.7281245$ (B3LYP / 6-31++G(D,P))

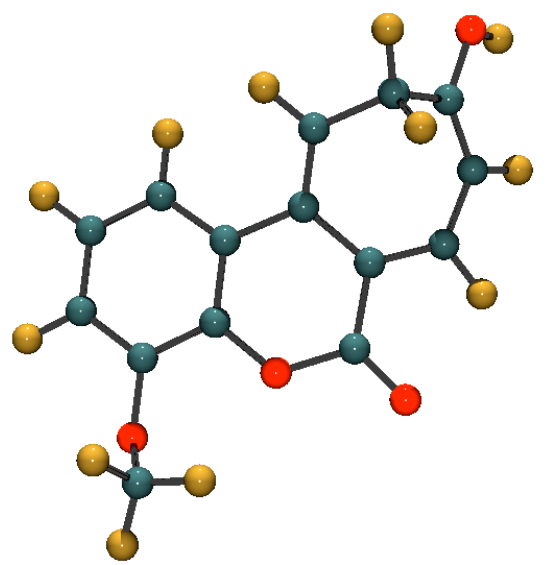

Standard orientation:

\begin{tabular}{|c|c|c|c|c|c|}
\hline \multirow{2}{*}{$\begin{array}{l}\text { Center } \\
\text { Number }\end{array}$} & \multirow{2}{*}{$\begin{array}{l}\text { Atomic } \\
\text { Number }\end{array}$} & \multirow{2}{*}{$\begin{array}{c}\text { Atomic } \\
\text { Type }\end{array}$} & \multicolumn{3}{|c|}{ Coordinates (Angstroms) } \\
\hline & & & $\mathrm{X}$ & Y & Z \\
\hline & - & & & --- & --------- \\
\hline 1 & 6 & 0 & 2.156982 & -2.741821 & -.383779 \\
\hline 2 & 6 & 0 & 3.197051 & -1.808231 & -.431506 \\
\hline 3 & 6 & 0 & 2.930417 & -.449733 & -.267431 \\
\hline 4 & 6 & 0 & 1.599094 & -.038200 & -.059659 \\
\hline 5 & 6 & 0 & .548367 & -.962563 & .022299 \\
\hline 6 & 6 & 0 & .850160 & -2.325031 & -.154006 \\
\hline 7 & 8 & 0 & 3.961138 & .442011 & -.401720 \\
\hline 8 & 6 & 0 & 4.288813 & 1.247751 & .742391 \\
\hline 9 & 8 & 0 & 1.394338 & 1.320254 & .068330 \\
\hline 10 & 6 & 0 & .141566 & 1.890041 & -.000246 \\
\hline 11 & 6 & 0 & -1.017614 & .970414 & -.032739 \\
\hline 12 & 6 & 0 & -.813478 & -.442862 & .281663 \\
\hline 13 & 8 & 0 & .081709 & 3.100619 & -.016327 \\
\hline 14 & 6 & 0 & -2.187066 & 1.510666 & -.525386 \\
\hline 15 & 6 & 0 & -3.434384 & .852616 & -.747499 \\
\hline 16 & 6 & 0 & -3.867534 & -.219079 & -.020772 \\
\hline 17 & 6 & 0 & -3.143094 & -.687464 & 1.206783 \\
\hline 18 & 6 & 0 & -1.807881 & -1.230920 & .769667 \\
\hline 19 & 8 & 0 & -5.001748 & -.908645 & -.293609 \\
\hline 20 & 1 & 0 & 2.370731 & -3.796165 & -.529112 \\
\hline 21 & 1 & 0 & 4.223597 & -2.110031 & -.611112 \\
\hline 22 & 1 & 0 & .051522 & -3.058214 & -.132446 \\
\hline 23 & 1 & 0 & 5.180076 & 1.809913 & .459434 \\
\hline 24 & 1 & 0 & 4.517963 & .608531 & 1.604126 \\
\hline 25 & 1 & 0 & 3.479310 & 1.938216 & .989882 \\
\hline 26 & 1 & 0 & -2.113743 & 2.541348 & -.863889 \\
\hline 27 & 1 & 0 & -4.088692 & 1.269367 & -1.511733 \\
\hline 28 & 1 & 0 & -3.735047 & -1.453220 & 1.711339 \\
\hline 29 & 1 & 0 & -3.009920 & .160082 & 1.892764 \\
\hline 30 & 1 & 0 & -1.643788 & -2.296794 & .889977 \\
\hline 31 & 1 & 0 & -5.363877 & -.640438 & -1.151309 \\
\hline
\end{tabular}

\begin{tabular}{lrr} 
& & \multicolumn{1}{c}{1} \\
& & \multicolumn{1}{c}{ A } \\
Frequencies -- & 44.2913 \\
Red. masses -- & 5.6905 \\
Frc consts -- & .0066 \\
IR Inten & -- & 1.7936
\end{tabular}

2
A
59.7389
2.0570
.0043
3.8444

3

A

70.4787

3.4938

.0102

.9854 
Theoretical data for compound $6 \mathrm{c}$ :

Final Energy in Hartrees: HF $=-879.2136405(B 3 L Y P / 6-31++G(D, P))$

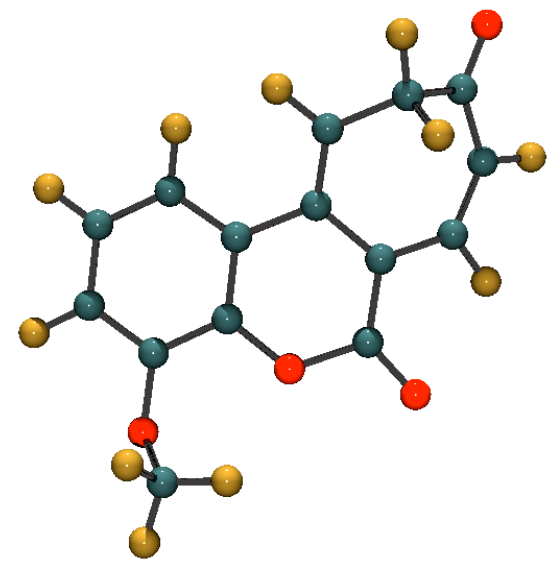

Standard orientation:

\begin{tabular}{|c|c|c|c|c|c|}
\hline \multirow{2}{*}{$\begin{array}{l}\text { Center } \\
\text { Number }\end{array}$} & \multirow{2}{*}{$\begin{array}{l}\text { Atomic } \\
\text { Number }\end{array}$} & \multirow{2}{*}{$\begin{array}{c}\text { Atomic } \\
\text { Type }\end{array}$} & \multicolumn{3}{|c|}{ Coordinates (Angstroms) } \\
\hline & & & $\mathrm{X}$ & $\mathrm{Y}$ & Z \\
\hline & & & -2 & -ー-ー-ー-ー-ー- & --------- \\
\hline 1 & 6 & 0 & 2.185309 & -2.715628 & -.451150 \\
\hline 2 & 6 & 0 & 3.217872 & -1.771403 & -.431789 \\
\hline 3 & 6 & 0 & 2.923821 & -.423348 & -.232175 \\
\hline 4 & 6 & 0 & 1.583825 & -.021499 & -.051986 \\
\hline 5 & 6 & 0 & .540493 & -.964032 & -.025046 \\
\hline 6 & 6 & 0 & .868203 & -2.315249 & -.244550 \\
\hline 7 & 8 & 0 & 3.964942 & .479378 & -.298058 \\
\hline 8 & 6 & 0 & 4.182045 & 1.301041 & .854313 \\
\hline 9 & 8 & 0 & 1.353882 & 1.316620 & .103115 \\
\hline 10 & 6 & 0 & .054628 & 1.868787 & -.057101 \\
\hline 11 & 6 & 0 & -1.051207 & .949796 & -.049098 \\
\hline 12 & 6 & 0 & -.829713 & -.466556 & .231270 \\
\hline 13 & 8 & 0 & .038906 & 3.088231 & -.168476 \\
\hline 14 & 6 & 0 & -2.285405 & 1.469457 & -.524359 \\
\hline 15 & 6 & 0 & -3.525127 & .871852 & -.677860 \\
\hline 16 & 6 & 0 & -4.016501 & -.305670 & -.006674 \\
\hline 17 & 6 & 0 & -3.154680 & -.817968 & 1.157600 \\
\hline 18 & 6 & 0 & -1.813531 & -1.295886 & .678651 \\
\hline 19 & 8 & 0 & -5.105635 & -.851162 & -.266622 \\
\hline 20 & 1 & 0 & 2.412757 & -3.763479 & -.628204 \\
\hline 21 & 1 & 0 & 4.254602 & -2.054259 & -.586565 \\
\hline 22 & 1 & 0 & .074104 & -3.053745 & -.268230 \\
\hline 23 & 1 & 0 & 5.095695 & 1.864704 & .649285 \\
\hline 24 & 1 & 0 & 4.333271 & .677344 & 1.746501 \\
\hline 25 & 1 & 0 & 3.348777 & 1.988128 & 1.018177 \\
\hline 26 & 1 & 0 & -2.197677 & 2.485931 & -.906055 \\
\hline 27 & 1 & 0 & -4.268355 & 1.394281 & -1.277253 \\
\hline 28 & 1 & 0 & -3.705123 & -1.625502 & 1.647432 \\
\hline 29 & 1 & 0 & -3.021895 & .003113 & 1.879168 \\
\hline 30 & 1 & 0 & -1.623113 & -2.363224 & .745801 \\
\hline
\end{tabular}

\begin{tabular}{|c|c|c|c|c|}
\hline & & 1 & 2 & 3 \\
\hline & & A & A & A \\
\hline Frequencies & -- & 45.9306 & 64.3066 & 76.7101 \\
\hline Red. masses & -- & 6.0821 & 4.1244 & 2.0953 \\
\hline Frc consts & -- & .0076 & .0100 & .0073 \\
\hline IR Inten & -- & .1810 & .4962 & 4.3992 \\
\hline
\end{tabular}


Theoretical data for compound $16 \mathrm{~b}$ :

Final Energy in Hartrees: HF $=-879.7239323(B 3 L Y P / 6-31++G(D, P))$

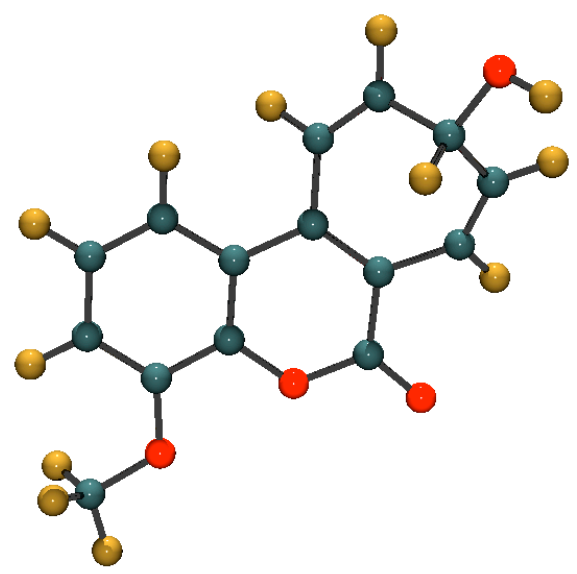

Standard orientation:

\begin{tabular}{|c|c|c|c|c|c|}
\hline \multirow{2}{*}{$\begin{array}{l}\text { Center } \\
\text { Number }\end{array}$} & \multirow{2}{*}{$\begin{array}{l}\text { Atomic } \\
\text { Number }\end{array}$} & \multirow{2}{*}{$\begin{array}{l}\text { Atomic } \\
\text { Type }\end{array}$} & \multicolumn{3}{|c|}{ Coordinates (Angstroms) } \\
\hline & & & $\mathrm{X}$ & $\mathrm{Y}$ & Z \\
\hline 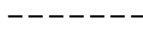 & -7 & & --------- & ---------- & ---------- \\
\hline 1 & 6 & 0 & 2.313636 & -2.524717 & -.034579 \\
\hline 2 & 6 & 0 & 3.287402 & -1.521575 & .099172 \\
\hline 3 & 6 & 0 & 2.910343 & -.179384 & .103795 \\
\hline 4 & 6 & 0 & 1.540311 & .140186 & -.039847 \\
\hline 5 & 6 & 0 & .562022 & -.850188 & -.191302 \\
\hline 6 & 6 & 0 & .973919 & -2.203104 & -.173513 \\
\hline 7 & 8 & 0 & 3.754516 & .875101 & .236832 \\
\hline 8 & 6 & 0 & 5.145914 & .612520 & .383549 \\
\hline 9 & 8 & 0 & 1.228656 & 1.467777 & -.018631 \\
\hline 10 & 6 & 0 & -.066495 & 1.926123 & -.115389 \\
\hline 11 & 6 & 0 & -1.143896 & .922561 & -.248391 \\
\hline 12 & 6 & 0 & -.835171 & -.431947 & -.327156 \\
\hline 13 & 8 & 0 & -.237690 & 3.126823 & -.095946 \\
\hline 14 & 6 & 0 & -2.461224 & 1.502454 & -.452011 \\
\hline 15 & 6 & 0 & -3.636450 & .915388 & -.146100 \\
\hline 16 & 6 & 0 & -3.710013 & -.397449 & .595603 \\
\hline 17 & 6 & 0 & -3.132057 & -1.428045 & -.328228 \\
\hline 18 & 6 & 0 & -1.828763 & -1.442453 & -.672601 \\
\hline 19 & 8 & 0 & -5.042575 & -.786329 & .918070 \\
\hline 20 & 1 & 0 & -5.402617 & -.160838 & 1.560309 \\
\hline 21 & 1 & 0 & -3.095492 & -.338933 & 1.508982 \\
\hline 22 & 1 & 0 & 5.620201 & 1.590181 & .471624 \\
\hline 23 & 1 & 0 & 5.547183 & .088860 & -.492954 \\
\hline 24 & 1 & 0 & 5.347403 & .025226 & 1.287983 \\
\hline 25 & 1 & 0 & 4.328959 & -1.798242 & .207039 \\
\hline 26 & 1 & 0 & 2.621108 & -3.565625 & -.020080 \\
\hline 27 & 1 & 0 & .236486 & -2.993098 & -.246137 \\
\hline 28 & 1 & 0 & -1.478040 & -2.262664 & -1.292258 \\
\hline 29 & 1 & 0 & -2.464268 & 2.502364 & -.875807 \\
\hline 30 & 1 & 0 & -3.821710 & -2.173319 & -.717202 \\
\hline 31 & 1 & 0 & -4.579352 & 1.376884 & -.432097 \\
\hline
\end{tabular}

\begin{tabular}{|c|c|c|c|c|}
\hline & & 1 & 2 & 3 \\
\hline & & A & A & A \\
\hline Frequencies & -- & 38.5875 & 70.4761 & 86.3899 \\
\hline Red. masses & -- & 5.0287 & 4.4451 & 4.4096 \\
\hline Frc consts & -- & .0044 & .0130 & .0194 \\
\hline IR Inten & -- & 1.2567 & 1.2303 & 7.4491 \\
\hline
\end{tabular}


Theoretical data for compound $16 \mathrm{c}$ :

Final Energy in Hartrees: HF $=-879.1417281(B 3 L Y P / 6-31++G(D, P))$

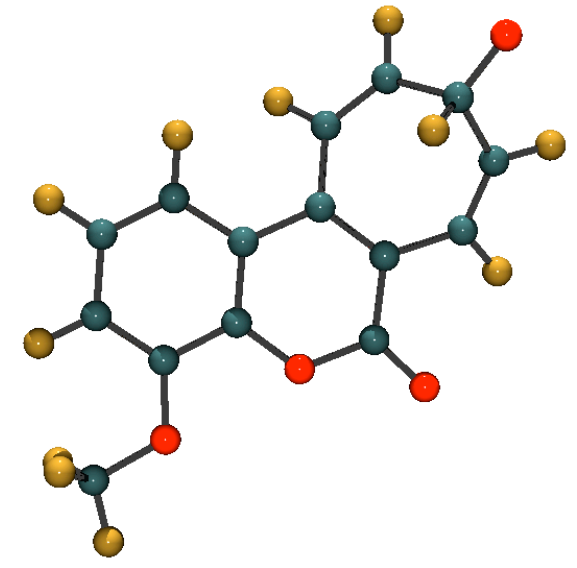

Standard orientation:

\begin{tabular}{|c|c|c|c|c|c|}
\hline \multirow{2}{*}{$\begin{array}{l}\text { Center } \\
\text { Number }\end{array}$} & \multirow{2}{*}{$\begin{array}{l}\text { Atomic } \\
\text { Number }\end{array}$} & \multirow{2}{*}{$\begin{array}{c}\text { Atomic } \\
\text { Type }\end{array}$} & \multicolumn{3}{|c|}{ Coordinates (Angstroms) } \\
\hline & & & $\mathrm{X}$ & Y & Z \\
\hline 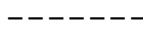 & & & ------- & ---------- & --------- \\
\hline 1 & 6 & 0 & 2.281582 & -2.524999 & -.035969 \\
\hline 2 & 6 & 0 & 3.261516 & -1.525346 & .089227 \\
\hline 3 & 6 & 0 & 2.878583 & -.184698 & .093915 \\
\hline 4 & 6 & 0 & 1.511165 & .142633 & -.042844 \\
\hline 5 & 6 & 0 & .525904 & -.843638 & -.185644 \\
\hline 6 & 6 & 0 & .942042 & -2.196024 & -.165001 \\
\hline 7 & 8 & 0 & 3.737053 & .873108 & .221239 \\
\hline 8 & 6 & 0 & 5.116740 & .594821 & .373757 \\
\hline 9 & 8 & 0 & 1.202955 & 1.469012 & -.024553 \\
\hline 10 & 6 & 0 & -.107562 & 1.928751 & -.112176 \\
\hline 11 & 6 & 0 & -1.184774 & .933225 & -.210600 \\
\hline 12 & 6 & 0 & -.874951 & -.430643 & -.306307 \\
\hline 13 & 8 & 0 & -.243814 & 3.138224 & -.114622 \\
\hline 14 & 6 & 0 & -2.506742 & 1.491459 & -.388863 \\
\hline 15 & 6 & 0 & -3.682335 & .871297 & -.103912 \\
\hline 16 & 6 & 0 & -3.860251 & -.452074 & .665997 \\
\hline 17 & 6 & 0 & -3.185835 & -1.396347 & -.342675 \\
\hline 18 & 6 & 0 & -1.864998 & -1.423881 & -.663443 \\
\hline 19 & 8 & 0 & -5.115891 & -.766033 & .983134 \\
\hline 20 & 1 & 0 & -3.142960 & -.385738 & 1.533909 \\
\hline 21 & 1 & 0 & 5.607119 & 1.565853 & .463721 \\
\hline 22 & 1 & 0 & 5.521859 & .063080 & -.498288 \\
\hline 23 & 1 & 0 & 5.312953 & .003492 & 1.278647 \\
\hline 24 & 1 & 0 & 4.303681 & -1.803029 & .192161 \\
\hline 25 & 1 & 0 & 2.583543 & -3.568496 & -.018447 \\
\hline 26 & 1 & 0 & .195891 & -2.979095 & -.225125 \\
\hline 27 & 1 & 0 & -1.504932 & -2.244669 & -1.282131 \\
\hline 28 & 1 & 0 & -2.523449 & 2.508271 & -.774003 \\
\hline 29 & 1 & 0 & -3.870579 & -2.116700 & -.792319 \\
\hline 30 & 1 & 0 & -4.626040 & 1.335332 & -.392471 \\
\hline
\end{tabular}

\begin{tabular}{|c|c|c|c|c|}
\hline & & 1 & 2 & 3 \\
\hline & & A & A & A \\
\hline Frequencies & -- & 37.4928 & 67.5092 & 80.6873 \\
\hline Red. masses & -- & 5.1564 & 4.8778 & 4.1550 \\
\hline Frc consts & -- & .0043 & .0131 & .0159 \\
\hline IR Inten & -- & 1.7583 & 3.0955 & 9.7902 \\
\hline
\end{tabular}


Theoretical data for compound 17a :

Final Energy in Hartrees: HF $=-879.7122644(B 3 L Y P / 6-31++G(D, P))$

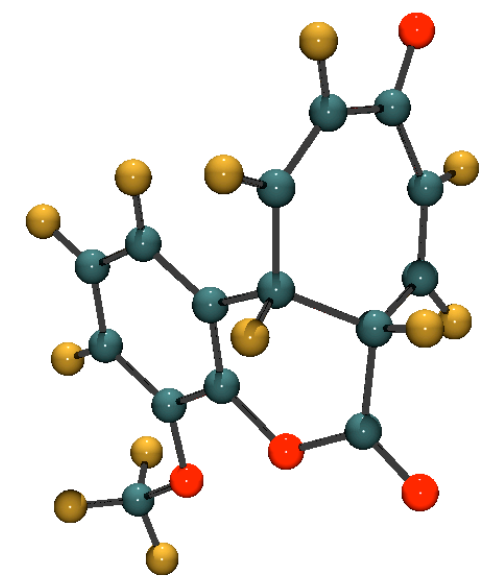

Standard orientation:

\begin{tabular}{|c|c|c|c|c|c|}
\hline \multirow{2}{*}{$\begin{array}{l}\text { Center } \\
\text { Number }\end{array}$} & \multirow{2}{*}{$\begin{array}{l}\text { Atomic } \\
\text { Number }\end{array}$} & \multirow{2}{*}{$\begin{array}{c}\text { Atomic } \\
\text { Type }\end{array}$} & \multicolumn{3}{|c|}{ Coordinates (Angstroms) } \\
\hline & & & $\mathrm{X}$ & $\mathrm{Y}$ & Z \\
\hline & & & - & ---------- & ---------- \\
\hline 1 & 6 & 0 & -1.407365 & -2.617793 & -.353510 \\
\hline 2 & 6 & 0 & -2.511180 & -1.883921 & .091888 \\
\hline 3 & 6 & 0 & -2.462644 & -.484794 & .108218 \\
\hline 4 & 6 & 0 & -1.287271 & .148718 & -.338407 \\
\hline 5 & 6 & 0 & -.185980 & -.577743 & -.788277 \\
\hline 6 & 6 & 0 & -.249333 & -1.976054 & -.787739 \\
\hline 7 & 8 & 0 & -3.465389 & .330273 & .524896 \\
\hline 8 & 6 & 0 & -4.678081 & -.263920 & .977631 \\
\hline 9 & 8 & 0 & -1.299485 & 1.539981 & -.344698 \\
\hline 10 & 6 & 0 & -.139932 & 2.263966 & -.352519 \\
\hline 11 & 6 & 0 & 1.164359 & 1.466933 & -.334175 \\
\hline 12 & 6 & 0 & 1.005148 & .244309 & -1.265935 \\
\hline 13 & 8 & 0 & -.200152 & 3.467952 & -.361076 \\
\hline 14 & 6 & 0 & 1.539576 & 1.167927 & 1.104427 \\
\hline 15 & 6 & 0 & 2.433881 & .279614 & 1.565513 \\
\hline 16 & 6 & 0 & 3.336691 & -.627133 & .816218 \\
\hline 17 & 6 & 0 & 3.248774 & -.862338 & -.648456 \\
\hline 18 & 6 & 0 & 2.270067 & -.540443 & -1.509397 \\
\hline 19 & 8 & 0 & 4.215835 & -1.221420 & 1.442351 \\
\hline 20 & 1 & 0 & .722069 & .661193 & -2.245052 \\
\hline 21 & 1 & 0 & 1.916690 & 2.147923 & -.750374 \\
\hline 22 & 1 & 0 & -5.326816 & .568478 & 1.251051 \\
\hline 23 & 1 & 0 & -4.507617 & -.898531 & 1.855855 \\
\hline 24 & 1 & 0 & -5.153477 & -.852466 & .183552 \\
\hline 25 & 1 & 0 & 4.088711 & -1.448926 & -1.012734 \\
\hline 26 & 1 & 0 & 2.607253 & .229950 & 2.637474 \\
\hline 27 & 1 & 0 & -3.399649 & -2.403535 & .429164 \\
\hline 28 & 1 & 0 & 2.386401 & -.900909 & -2.530410 \\
\hline 29 & 1 & 0 & -1.456377 & -3.702220 & -.353650 \\
\hline 30 & 1 & 0 & .606695 & -2.554914 & -1.116441 \\
\hline 31 & 1 & 0 & 1.049379 & 1.795701 & 1.846893 \\
\hline
\end{tabular}

\begin{tabular}{|c|c|c|c|c|}
\hline & & 1 & 2 & 3 \\
\hline & & A & A & A \\
\hline Frequencies & -- & 25.4191 & 48.0998 & 70.2939 \\
\hline Red. masses & -- & 8.2299 & 4.7776 & 4.2892 \\
\hline Frc consts & -- & .0031 & .0065 & .0125 \\
\hline IR Inten & -- & 4.0869 & 1.8111 & 2.6267 \\
\hline
\end{tabular}

